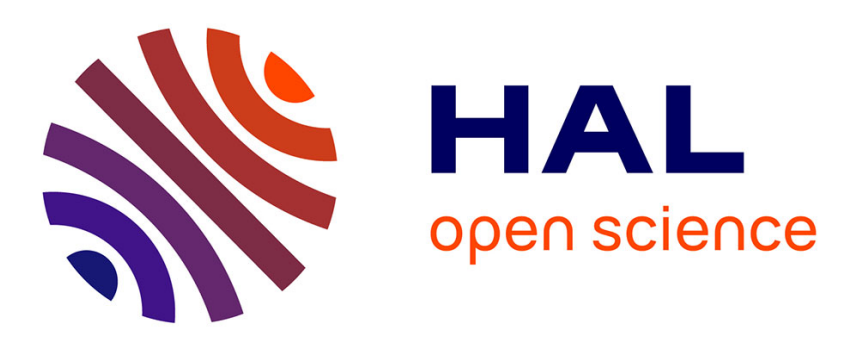

\title{
Impact of Strong Magnetic Fields on Collision Mechanism for Transport of Charged Particles
}

Mihai Bostan, Irene Gamba

\section{To cite this version:}

Mihai Bostan, Irene Gamba. Impact of Strong Magnetic Fields on Collision Mechanism for Transport of Charged Particles. Journal of Statistical Physics, 2012, 148 (5), pp.856-895. 10.1007/s10955-0120560-4 . hal-01266547

\section{HAL Id: hal-01266547 https://hal.science/hal-01266547}

Submitted on 2 Feb 2016

HAL is a multi-disciplinary open access archive for the deposit and dissemination of scientific research documents, whether they are published or not. The documents may come from teaching and research institutions in France or abroad, or from public or private research centers.
L'archive ouverte pluridisciplinaire HAL, est destinée au dépôt et à la diffusion de documents scientifiques de niveau recherche, publiés ou non, émanant des établissements d'enseignement et de recherche français ou étrangers, des laboratoires publics ou privés. 


\title{
Impact of strong magnetic fields on collision mechanism for transport of charged particles
}

\author{
Mihai Bostan *, Irene M. Gamba ${ }^{\dagger}$
}

(July 27, 2012)

\begin{abstract}
One of the main applications in plasma physics concerns the energy production through thermo-nuclear fusion. The controlled fusion is achieved by magnetic confinement i.e., the plasma is confined into a toroidal domain (tokamak) under the action of huge magnetic fields. Several models exist for describing the evolution of strongly magnetized plasmas, most of them by neglecting the collisions between particles. The subject matter of this paper is to investigate the effect of large magnetic fields with respect to a collision mechanism. We consider here linear collision Boltzmann operators and derive, by averaging with respect to the fast cyclotronic motion due to strong magnetic forces, their effective collision kernels.
\end{abstract}

Keywords: Linear Boltzmann equation, Multiple scales, Average operator.

AMS classification: 35Q75, 78A35, 82D10.

\section{Introduction}

The goal of this paper is to emphasize the role of the collision mechanism in the framework of magnetic confinement. We investigate both first and second order asymptotic approximations, when the magnetic field becomes large. We present completely explicit models and clearly indicate the form of the effective collision operator, the expression of the collision drift and check that the standard physical properties hold true.

Motivated by the magnetic fusion, many research programs in plasma physics concern the dynamics of charged particles under the action of strong magnetic fields $\left|\mathbf{B}^{\varepsilon}\right| \rightarrow+\infty$ as $\varepsilon \searrow 0$. Usually we appeal to kinetic models meaning that we replace the particles by their density (distribution) function $f^{\varepsilon}=f^{\varepsilon}(t, x, v)$ over the phase space, depending on time $t \in \mathbb{R}_{+}$, position $x \in \mathbb{R}^{3}$ and velocity $v \in \mathbb{R}^{3}$. The quantity $f^{\varepsilon}(t, x, v) \mathrm{d} x \mathrm{~d} v$ represents the particle number at time $t$, inside the infinitesimal volume $\mathrm{d} x \mathrm{~d} v$ around $(x, v)$. The fluctuations of the density $f^{\varepsilon}$ are due to the transport of the charged particles under the electro-magnetic field and also to the collisions between particles

$$
\partial_{t} f^{\varepsilon}+v \cdot \nabla_{x} f^{\varepsilon}+\frac{q}{m}\left(E(x)+v \wedge \mathbf{B}^{\varepsilon}(x)\right) \cdot \nabla_{v} f^{\varepsilon}=Q\left(f^{\varepsilon}\right), \quad(t, x, v) \in \mathbb{R}_{+} \times \mathbb{R}^{3} \times \mathbb{R}^{3} .
$$

*Laboratoire d'Analyse, Topologie, Probabilités LATP, Centre de Mathématiques et Informatique CMI, UMR CNRS 7353, 39 rue Frédéric Joliot Curie, 13453 Marseille Cedex 13 France. E-mail : bostan@cmi.univ-mrs.fr

${ }^{\dagger}$ ICES \& Department of Mathematics, University of Texas at Austin, Texas 78712 USA. E-mail: gamba@math.utexas.edu 
We add the initial condition

$$
f^{\varepsilon}(0, x, v)=f^{\text {in }}(x, v), \quad(x, v) \in \mathbb{R}^{3} \times \mathbb{R}^{3} .
$$

Here $m$ is the particle mass and $q$ is the particle charge, $\left(E, \mathbf{B}^{\varepsilon}\right)$ stands for the electro-magnetic field and $Q$ is the collision operator. We neglect the self-consistent electro-magnetic field; we assume that the magnetic field is stationary, divergence free and that the electric field derives from a given electric potential $E(x)=-\nabla_{x} \phi(x)$. We focus on the linear Boltzmann collision operator

$$
Q(f)(v)=\frac{1}{\tau} \int_{\mathbb{R}^{3}} s\left(v, v^{\prime}\right)\left\{M(v) f\left(v^{\prime}\right)-M\left(v^{\prime}\right) f(v)\right\} \mathrm{d} v^{\prime}
$$

where $M$ is the Maxwellian equilibrium

$$
M(v)=\frac{1}{(2 \pi \theta / m)^{3 / 2}} \exp \left(-\frac{m|v|^{2}}{2 \theta}\right)
$$

with temperature $\theta>0, \tau$ is the relaxation time and $s$ is the scattering cross section. We assume that

$$
s\left(v, v^{\prime}\right)=s\left(v^{\prime}, v\right), \quad v, v^{\prime} \in \mathbb{R}^{3}
$$

and

$$
s_{0} \leq s\left(v, v^{\prime}\right) \leq S_{0}, \quad v, v^{\prime} \in \mathbb{R}^{3}
$$

for some $s_{0}, S_{0}>0$. We analyze the asymptotic behaviour of (1) when the magnetic field becomes large

$$
\mathbf{B}^{\varepsilon}(x)=\frac{\mathbf{B}(x)}{\varepsilon}, \quad \mathbf{B}(x)=B(x) b(x), \operatorname{div}_{x}(B b)=0, \quad 0<\varepsilon<<1
$$

for some scalar positive function $B(x)$ and some field of unitary vectors $b(x)$.

These studies are motivated by the numerical simulations of strongly magnetized plasmas. Indeed, a direct resolution of (1) using explicit numerical schemes requires a time discretization of order $\mathcal{O}\left(1 /\left|\mathbf{B}^{\varepsilon}\right|\right)=\mathcal{O}(\varepsilon)$, and therefore a huge computation time, when $\varepsilon$ becomes very small. The main idea will be to perform the asymptotic analysis of (1) at the theoretical level i.e., to replace (1) by first/second order approximation models whose coefficients are stable when $\varepsilon \searrow 0$. Eventually we solve numerically these asymptotic models. Notice that in this case the largest time step satisfying the stability condition does not depend on $\varepsilon$, and therefore the numerical resolution remains efficient even for very large magnetic fields.

For simplicity we neglect the magnetic curvature i.e., $\partial_{x} b=0$. Assuming that $b=e_{3}$ and taking into account the divergence constraint in (6) lead to $\mathbf{B}^{\varepsilon}(x)=\left(0,0, B^{\varepsilon}\left(x_{1}, x_{2}\right)\right)=$ $\left(0,0, B\left(x_{1}, x_{2}\right) / \varepsilon\right)$. Therefore the equation (1) should be understood in two dimensional setting

$$
\partial_{t} f^{\varepsilon}+v \cdot \nabla_{x} f^{\varepsilon}+\frac{q}{m}\left(E(x)+\frac{{ }^{\perp} v}{\varepsilon} B(x)\right) \cdot \nabla_{v} f^{\varepsilon}=Q\left(f^{\varepsilon}\right), \quad t>0,(x, v) \in \mathbb{R}^{2} \times \mathbb{R}^{2}
$$

where ${ }^{\perp} v=\left(v_{2},-v_{1}\right)$ and $Q$ is the two dimensional linear Boltzmann operator, associated to the Maxwellian $M(v)=m /(2 \pi \theta) \exp \left(-\frac{m|v|^{2}}{2 \theta}\right), v \in \mathbb{R}^{2}$. In the sequel we summarize the main results of the paper.

The dynamics of the particles is dominated by the transport operator $\frac{1}{\varepsilon} \mathcal{T}$ where

$$
\mathcal{T}=\frac{q B(x)}{m}{ }^{\perp} v \cdot \nabla_{v} .
$$


When $\varepsilon$ is small enough it is natural to introduce expansions like $f^{\varepsilon}=f+\varepsilon f^{1}+\varepsilon^{2} f^{2}+\ldots$ Plugging the previous Ansatz in (7) gives at the lowest order the divergence constraint $\mathcal{T} f=0$ and to the next order the evolution equation

$$
\partial_{t} f+v \cdot \nabla_{x} f+\frac{q}{m} E(x) \cdot \nabla_{v} f+\mathcal{T} f^{1}=Q(f) .
$$

We expect that the Boltzmann equation (9) supplemented by the constraint $\mathcal{T} f=0$ (and also by an appropriate initial condition) provides a well posed problem for the leading order term $f$.

The approach is standard as in the Hilbert expansion methods of matching orders of $\varepsilon$ : the first order term $f^{1}$ enters (9) as a Lagrange multiplier in such a way that at any time $t>0$ the constraint $\mathcal{T} f(t)=0$ should be satisfied. Finding a closure for the dominant term $f$ reduces to eliminating the Lagrange multiplier provided that the constraint on $f$ holds true. Notice that the range and kernel of the operator $\mathcal{T}$ are orthogonal (in $L^{2}$ ) and therefore the closure for $f$ follows naturally by taking the orthogonal projection on $\operatorname{ker} \mathcal{T}$ of the Boltzmann equation (9) at any fixed time $t>0$. One of the key point is to observe that taking the orthogonal projection on the kernel of $\mathcal{T}$ reduces to averaging along the flow associated to $\mathcal{T}$ cf. [3], [4], [5]. Observe that the motion associated to the vector field $\left(0,0, \omega_{c}(x)^{\perp} v\right)$ is periodic

$$
X(s ; x, v)=x, \quad V(s ; x, v)=R\left(-\omega_{c}(x) s\right) v
$$

where $\omega_{c}(x)=q B(x) / m$ is the rescaled cyclotronic frequency and for any $\alpha \in \mathbb{R}$ the notation $R(\alpha)$ stands for the rotation of angle $\alpha$

$$
R(\alpha)=\left(\begin{array}{rr}
\cos \alpha & -\sin \alpha \\
\sin \alpha & \cos \alpha
\end{array}\right)
$$

We introduce the rescaled cyclotronic period $T_{c}(x)=2 \pi / \omega_{c}(x)$. In this case, for any function $u \in L^{2}\left(\mathbb{R}^{2} \times \mathbb{R}^{2}\right)$, the average simply writes cf. [4]

$$
\begin{aligned}
\langle u\rangle(x, v) & =\frac{1}{T_{c}(x)} \int_{0}^{T_{c}(x)} u(X(s ; x, v), V(s ; x, v)) \mathrm{d} s \\
& =\frac{1}{2 \pi} \int_{0}^{2 \pi} u(x, R(-\alpha) v) \mathrm{d} \alpha=\frac{1}{2 \pi} \int_{0}^{2 \pi} u(x, R(\alpha) v) \mathrm{d} \alpha .
\end{aligned}
$$

Applying the average operator to the Boltzmann equation (9) yields the following closure for the dominant term $f$

$$
\left\langle\partial_{t} f\right\rangle+\left\langle v \cdot \nabla_{x} f+\frac{q}{m} E(x) \cdot \nabla_{v} f\right\rangle=\langle Q(f)\rangle
$$

supplemented by the constraint $\mathcal{T} f(t)=0$ at any time $t>0$. Notice that

$$
\psi_{1}=x_{1}, \quad \psi_{2}=x_{2}, \quad \psi_{3}=|v|
$$

form a complete family of prime integrals for $\mathcal{T}$ and thus the constraint $\mathcal{T} f=0$ reads $f(t, x, v)=g(t, x, r=|v|)$ for some function $g=g(t, x, r)$. Straightforward computations show that

$$
\left\langle\partial_{t} f\right\rangle=\partial_{t}\langle f\rangle=\partial_{t} f,\left\langle v \cdot \nabla_{x} f\right\rangle=\left\langle v \cdot \nabla_{x} g(t, x,|v|)\right\rangle=0,\left\langle E \cdot \nabla_{v} f\right\rangle=\left\langle E \cdot \frac{v}{|v|} \partial_{r} g\right\rangle=0 .
$$

Moreover, we will see that the linear collision operator commutes with the average operator i.e., $\langle Q(f)\rangle=Q(\langle f\rangle)=Q(f)$ and finally the dominant term satisfies the space homogeneous Boltzmann equation [12], [15], [25]

$$
\partial_{t} f=Q(f)
$$


In particular there is no current $\int_{\mathbb{R}^{2}} v f(t, x, v) \mathrm{d} v=\int_{\mathbb{R}^{2}} v g(t, x,|v|) \mathrm{d} v=0$ along the orthogonal directions to the magnetic lines, as expected by the magnetic confinement context. But orthogonal drifts may appear at the next order. It is well known that the particles move slowly along the orthogonal directions and we distinguish the electric cross field drift $v_{\wedge}^{\varepsilon}=\frac{\perp_{E}}{B^{\varepsilon}}$, the magnetic gradient drift $v_{\mathrm{GD}}^{\varepsilon}=-\frac{|v|^{2}}{2 \omega_{\varepsilon}^{\varepsilon}} \frac{{ }^{\perp} \nabla_{x} B^{\varepsilon}}{B^{\varepsilon}}, \omega_{c}^{\varepsilon}=\frac{\omega_{c}}{\varepsilon}$, the magnetic curvature drift (which is neglected here since $\partial_{x} b=0$ ) cf. [27]. We intend to investigate the effect of strong magnetic fields through the collision mechanism. We will also prove that the collisions account for a drift motion which, to the best of our knowledge, has not been reported yet. As calculated in Corollary 4.1, this effect is proven to be generic for scattering cross-sections satisfying conditions (4) and (5). However, in the case of a constant scattering cross section $s$ (i.e. $s_{1}=s$ and $s_{2}=0$ ) we find a reduced expression with the explicit formula

$$
v_{\mathrm{QD}}^{\varepsilon}=-\frac{s}{\tau} \frac{\perp v}{\omega_{c}^{\varepsilon}} .
$$

Naturally, in order to observe the previous drifts (of order of $\varepsilon$ ) we need to take into account the first order correction term in the expansion $f^{\varepsilon}=f+\varepsilon f^{1}+\varepsilon^{2} f^{2}+\ldots$ eventually to appeal to the next order evolution equation

$$
\partial_{t} f^{1}+v \cdot \nabla_{x} f^{1}+\frac{q}{m} E \cdot \nabla_{v} f^{1}+\mathcal{T} f^{2}=Q\left(f^{1}\right) .
$$

As before we are looking for a closure with respect to $f+\varepsilon f^{1}$, by getting rid of the second order correction $f^{2}$. This will be achieved using again the averaging technique and combining properly the time evolutions of $f, f^{1}$ in $(9),(13)$. Doing that we will get another Boltzmann like equation, whose collision operator $Q+\varepsilon Q^{1}$ is a first order perturbation of the standard linear Boltzmann operator $Q$. For example, when the scattering cross section is constant we obtain

$$
\varepsilon Q^{1}(f)(x, v)=\frac{s}{\tau} M(v)\left\{m \frac{v \cdot v_{\wedge}^{\varepsilon}}{\theta} \rho_{f}(x)-\frac{v}{\omega_{c}^{\varepsilon}} \cdot{ }^{\perp} \nabla_{x} \rho_{f}+\operatorname{div}_{x}\left(\frac{{ }^{\perp} j_{f}}{\omega_{c}^{\varepsilon}}\right)\right\}
$$

where $\rho_{f}(x)=\int_{\mathbb{R}^{2}} f\left(x, v^{\prime}\right) \mathrm{d} v^{\prime}, j_{f}(x)=\int_{\mathbb{R}^{2}} v^{\prime} f\left(x, v^{\prime}\right) \mathrm{d} v^{\prime}$. Surely the construction of the new collision operator should take into account the classical physical properties, as mass conservation and entropy inequality. We will see that $\varepsilon Q^{1}$ (and therefore $Q+\varepsilon Q^{1}$ as well) satisfies global (in space and velocity) mass conservation and entropy inequality.

We point out that a linearized and gyroaveraged collision operator has been written in [29], but the implementation of this operator seems very hard. We refer to [10] for a general guiding-center bilinear Fokker-Planck collision operator. Another difficulty lies in the relaxation of the distribution function towards a local Maxwellian equilibrium. Most of the available model operators, in particular those which are linearized near a Maxwellian, are missing this property. Very recently a set of model collision operators has been obtained in [17], based on entropy variational principles.

The analysis of the Vlasov or Vlasov-Poisson equations with large external magnetic field have been carried out in [16], [20]. The numerical approximation of the gyrokinetic models has been performed in [21] using semi-Lagrangian schemes, see also [18], [19]. For recent results on the Boltzmann equation we refer to [7], [8]. High electric field limits of the Boltzmann-Poisson system have been addressed in [26], [13], [14], [1].

The nonlinear gyrokinetic theory of the Vlasov-Maxwell equations can be carried out by appealing to Lagrangian and Hamiltonian methods [11], [23], [24]. It is also possible to follow the general method of multiple time scale or averaging perturbation developped in [2]. For a unified treatment of the main physical ideas and theoretical methods that have emerged on magnetic plasma confinement we refer to [22]. 
The outline of the paper is the following. In Section 2 we introduce our models and present the main results. Section 3 is devoted to the definition and main properties of the average operator. In particular we investigate its commutation properties with respect to first order differential operators and the collision operator. The first and second order asymptotic models for the linear Boltzmann equation with large magnetic field are detailed in Section 4.

\section{Presentation of the models and main results}

The main results are stated in this section, the proofs being postponed to the next sections. As observed formally before, the first order approximation of (7) leads to the space homogeneous Boltzmann equation. We obtain rigorous weak and strong convergence results, at least for electric potential satisfying

$$
E(x)=-\nabla_{x} \phi(x), \quad \int_{\mathbb{R}^{2}} \exp \left(-q \frac{\phi(x)}{\theta}\right) \mathrm{d} x<+\infty .
$$

The equilibrium

$$
F(x, v)=M(v) \exp (-q \phi(x) / \theta), \quad(x, v) \in \mathbb{R}^{2} \times \mathbb{R}^{2}
$$

belongs to $L_{F}^{2}=L^{2}\left(\frac{\mathrm{d} v \mathrm{~d} x}{F(x, v)}\right)$ and by standard computations we obtain the uniform bound

$$
\int_{\mathbb{R}^{2}} \int_{\mathbb{R}^{2}} \frac{\left(f^{\varepsilon}(t, x, v)\right)^{2}}{F(x, v)} \mathrm{d} v \mathrm{~d} x \leq \int_{\mathbb{R}^{2}} \int_{\mathbb{R}^{2}} \frac{\left(f^{\mathrm{in}}(x, v)\right)^{2}}{F(x, v)} \mathrm{d} v \mathrm{~d} x, \quad \varepsilon>0
$$

which allows us to get weak compactness of $\left(f^{\varepsilon}\right)_{\varepsilon>0}$ in $L^{\infty}\left(\mathbb{R}_{+} ; L_{F}^{2}\right)$. For scattering cross section of the form $s\left(v, v^{\prime}\right)=\sigma\left(\left|v-v^{\prime}\right|\right)$ one has (see Section 4.1 for proof details)

Theorem 2.1 Assume that the scattering cross section has the form $s\left(v, v^{\prime}\right)=\sigma\left(\left|v-v^{\prime}\right|\right) \in$ $\left[s_{0}, S_{0}\right], v, v^{\prime} \in \mathbb{R}^{2}$ and that the electrostatic potential verifies (14). For any $\varepsilon>0$ we denote by $f^{\varepsilon}$ the weak solution of (7), (2) associated to a initial condition satisfying $f^{\text {in }} \in L_{F}^{2}$. Therefore $\left(f^{\varepsilon}\right)_{\varepsilon>0}$ converges weakly $\star$ in $L^{\infty}\left(\mathbb{R}_{+} ; L_{F}^{2}\right)$ as $\varepsilon \searrow 0$ towards the weak solution of the problem

$$
\partial_{t} f=Q(f), \quad(t, x, v) \in \mathbb{R}_{+}^{\star} \times \mathbb{R}^{2} \times \mathbb{R}^{2}, \quad f(0, x, v)=\left\langle f^{\text {in }}\right\rangle(x, v), \quad(x, v) \in \mathbb{R}^{2} \times \mathbb{R}^{2} .
$$

Under additional smoothness hypotheses, strong convergence results hold true as well. The main idea is to introduce a smooth first order corrector (see Section 4.1)

Theorem 2.2 Assume that the scattering cross section satisfies $s\left(v, v^{\prime}\right)=\sigma\left(\left|v-v^{\prime}\right|\right) \in$ $\left[s_{0}, S_{0}\right], v, v^{\prime} \in \mathbb{R}^{2}$ for some function $\sigma \in W^{2, \infty}$, that the electro-magnetic field $(E(x), B(x))$ belongs to $W^{1, \infty}\left(\mathbb{R}^{2}\right)$ such that (14) holds true and the magnetic field is bounded from below

$$
\inf _{x \in \mathbb{R}^{2}} B(x)>0
$$

and that the initial condition verifies

$$
\mathcal{T} f^{\text {in }}=0, \quad\left(1+|v|^{2}\right)\left|\nabla_{x, v}^{2} f^{\text {in }}\right| \in L_{F}^{2} .
$$

Then for any $T>0$ there is a constant $C_{T}$ such that

$$
\left\|f^{\varepsilon}(t)-f(t)\right\|_{L_{F}^{2}} \leq C_{T} \varepsilon, \quad t \in[0, T], \quad \varepsilon>0 .
$$


The leading order term in the expansion of $f^{\varepsilon}$ satisfies, at any fixed space point, the space homogeneous Boltzmann equation. There is no phase space transport, the collision operator $Q$ is local in space and there is no current, since $f(t, x, \cdot)$ depends only on $|v|$. A much more interesting model will be the second order approximation, since we expect to retrieve the usual drifts along the directions orthogonal to the magnetic lines, but also a collision drift. Certainly, the second order approximation will appear as a first order perturbation of the space homogeneous Boltzmann equation, involving a new transport operator but also a new collision operator $Q+\varepsilon Q^{1}$. The full expression of the operator $Q^{1}$ is rather complicated, see Corollary 4.1, but it simplifies a lot when the scattering cross section is constant

$$
Q^{1}(f)=\frac{s}{\tau} M(v)\left[\operatorname{div}_{x}\left(\frac{{ }^{\perp} j_{f}(x)}{\omega_{c}(x)}\right)+\frac{\rho_{F}(x)}{\omega_{c}(x)}{ }^{\perp} v \cdot \nabla_{x}\left(\frac{\rho_{f}(x)}{\rho_{F}(x)}\right)\right]
$$

where $\rho_{f}=\int_{\mathbb{R}^{2}} f \mathrm{~d} v^{\prime}, \rho_{F}=\int_{\mathbb{R}^{2}} F \mathrm{~d} v^{\prime}, j_{f}=\int_{\mathbb{R}^{2}} v^{\prime} f \mathrm{~d} v^{\prime}$. In this case the collision operator to be considerer is (see (19))

$$
\left(Q+\varepsilon Q^{1}\right)(f)=\frac{s}{\tau} M(v)\left(\rho_{f}-\frac{f}{M(v)}\right)+\varepsilon \frac{s}{\tau} M(v)\left[\operatorname{div}_{x}\left(\frac{{ }^{\perp} j_{f}(x)}{\omega_{c}(x)}\right)+\frac{\rho_{F}(x)}{\omega_{c}(x)}{ }^{\perp} v \cdot \nabla_{x}\left(\frac{\rho_{f}(x)}{\rho_{F}(x)}\right)\right]
$$

and satisfies only a global mass balance i.e., $\int_{\mathbb{R}^{2}} \int_{\mathbb{R}^{2}}\left\{Q(f)+\varepsilon Q^{1}(f)\right\} \mathrm{d} v \mathrm{~d} x=0$. For the sake of the presentation we formulate the second order convergence result in the context of constant scattering cross section but we point out that similar results hold true in the general case (see Section 4.2 for proof details).

Theorem 2.3 Assume that the electro-magnetic field $\left(E(x), B^{\varepsilon}(x)=B(x) / \varepsilon\right)$ is smooth such that (14) and (18) hold true. We denote by $f^{\varepsilon}$ the weak solution of (7) with the initial condition $f^{\varepsilon}(0)=f_{\text {in }}^{\varepsilon}$ and by $\tilde{f}^{\varepsilon}$ the weak solution of

$$
\begin{aligned}
& \partial_{t} \tilde{f}^{\varepsilon}+\left(\frac{{ }^{\perp} E}{B^{\varepsilon}}-\frac{|v|^{2}}{2 \omega_{c}^{\varepsilon}} \frac{{ }^{\perp} \nabla_{x} B^{\varepsilon}}{B^{\varepsilon}}\right) \cdot \nabla_{x} \tilde{f}^{\varepsilon}+\left(\frac{{ }^{\perp} E}{2 B^{\varepsilon}} \cdot \frac{\nabla_{x} B^{\varepsilon}}{B^{\varepsilon}}\right) v \cdot \nabla_{v} \tilde{f}^{\varepsilon} \\
& =\frac{s}{\tau} M(v)\left(\rho_{\tilde{f}^{\varepsilon}}-\frac{\tilde{f}^{\varepsilon}}{M(v)}-\frac{\rho_{F}}{\omega_{c}^{\varepsilon}} v \cdot{ }^{\perp} \nabla_{x}\left(\frac{\rho_{\tilde{f}^{\varepsilon}}}{\rho_{F}}\right)+\operatorname{div}_{x}\left(\frac{{ }^{\perp} j_{\tilde{f}^{\varepsilon}}}{\omega_{c}^{\varepsilon}}\right)\right)
\end{aligned}
$$

with the initial condition

$$
\tilde{f}^{\varepsilon}(0)=f^{\text {in }}+\frac{{ }^{\perp} v}{\omega_{c}^{\varepsilon}} \cdot \nabla_{x} f^{\text {in }}-\frac{{ }^{\perp} E}{B^{\varepsilon}} \cdot \nabla_{v} f^{\text {in }}, \mathcal{T} f^{\text {in }}=0
$$

where $\omega_{c}^{\varepsilon}=\frac{q B^{\varepsilon}}{m}=\frac{\omega_{c}}{\varepsilon}$ is the cyclotronic frequency. If the family of the initial conditions $\left(f_{\text {in }}^{\varepsilon}\right)_{\varepsilon>0}$ satisfies

$$
f_{\text {in }}^{\varepsilon}=f^{\text {in }}+\frac{{ } v}{\omega_{c}^{\varepsilon}} \cdot \nabla_{x} f^{\text {in }}-\frac{{ }^{\perp} E}{B^{\varepsilon}} \cdot \nabla_{v} f^{\text {in }}+\mathcal{O}\left(\varepsilon^{2}\right)
$$

then we have $f^{\varepsilon}=\tilde{f}^{\varepsilon}+\mathcal{O}\left(\varepsilon^{2}\right)$.

Remark 2.1 Notice that the coefficients in (19) still depend on $\varepsilon$ but they remain of order $\mathcal{O}(1)$ or $\mathcal{O}(\varepsilon)$ when $\varepsilon \searrow 0$. In particular the numerical approximation can be achieved uniformly with respect to $\varepsilon>0$, using a time step not depending on $\varepsilon$. 


\section{Averaging along a characteristic flow}

This section is devoted to the average technique. It consists in averaging along the characteristic flow associated to a divergence free vector field. When applied to the gyrokinetic theory, this method is known as the gyroaverage technique. It allows us to derive limit models by neglecting the fluctuations associated to fast motions, as the cyclotron rotation of particles around the magnetic lines. From the mathematical point of view, it reduces to averaging along the characteristic flow associated to the dominant advection field (here the magnetic force in the framework of magnetic confinement) and it is also equivalent to projecting onto the subspace of functions which are left invariant by the same flow. These tools are well known and have been used when establishing the guiding center approximation in three dimensions for general magnetic shapes [5], the finite Larmor radius regime [3] and, more generally, when studying transport equations $\mathrm{w}$ ith disparate advection fields [4]. We point out that the averaging technique applies to many other cases : models which take into account the mass ratio between electrons and ions subject to strong magnetic fields [9] and models with fast oscillating magnetic fields [6]. In the present work we generalize the averaging method to collisional models. Motivated by the study of the Boltzmann equation, we revisit some results concerning the average of first order differential operators which will combine with other new results in order to study the average of collision operators.

\subsection{Gyroverage operator}

We recall the definition and the properties of the average operator corresponding to the transport operator $\mathcal{T}$ given in (8), now in divergence form, whose definition in the $L^{2}\left(\mathbb{R}^{2} \times \mathbb{R}^{2}\right)$ setting is given by

$$
\mathcal{T} u=\operatorname{div}_{v}\left(\omega_{c}(x) u(x, v){ }^{\perp} v\right), \omega_{c}(x)=\frac{q B(x)}{m}
$$

for any function $u$ in the domain

$$
\mathrm{D}(\mathcal{T})=\left\{u(x, v) \in L^{2}\left(\mathbb{R}^{2} \times \mathbb{R}^{2}\right): \operatorname{div}_{v}\left(\omega_{c}(x) u^{\perp} v\right) \in L^{2}\left(\mathbb{R}^{2} \times \mathbb{R}^{2}\right)\right\} .
$$

We denote by $\|\cdot\|$ the standard norm of $L^{2}\left(\mathbb{R}^{2} \times \mathbb{R}^{2}\right)$ and by $(X, V)(s ; x, v)$ the characteristics associated to $\omega_{c}(x)^{\perp} v \cdot \nabla_{v}$

$$
\frac{\mathrm{d} X}{\mathrm{~d} s}=0, \quad \frac{\mathrm{d} V}{\mathrm{~d} s}=\omega_{c}(X(s)){ }^{\perp} V(s), \quad(X, V)(0)=(x, v) .
$$

A complete family of invariants for the flow (10) is given by (12) and therefore the kernel of $\mathcal{T}$ is given by those functions in $L^{2}\left(\mathbb{R}^{2} \times \mathbb{R}^{2}\right)$ depending only on $x_{1}, x_{2}$ and $|v|$. The following two results are justified in [5]. The first one states that averaging reduces to orthogonal projection onto the kernel of $\mathcal{T}$ (cf. [5] Proposition 2.1). The second one concerns the invertibility of $\mathcal{T}$ on the subspace of zero average functions and establishes a Poincaré inequality.

Proposition 3.1 The average operator is linear continuous. Moreover it coincides with the orthogonal projection on the kernel of $\mathcal{T}$ i.e.,

$$
\langle u\rangle \in \operatorname{ker} \mathcal{T} \text { and } \int_{\mathbb{R}^{2}} \int_{\mathbb{R}^{2}}(u-\langle u\rangle) \varphi \mathrm{d} v \mathrm{~d} x=0, \forall \varphi \in \operatorname{ker} \mathcal{T} .
$$

Remark 3.1 Notice that we have the orthogonal decomposition of $L^{2}\left(\mathbb{R}^{2} \times \mathbb{R}^{2}\right)$ into invariant functions along the characteristics (10) and zero average functions

$$
u=\langle u\rangle+(u-\langle u\rangle), \quad \int_{\mathbb{R}^{2}} \int_{\mathbb{R}^{2}}(u-\langle u\rangle)\langle u\rangle \mathrm{d} v \mathrm{~d} x=0 .
$$


Taking into account that $\mathcal{T}^{\star}=-\mathcal{T}$, the equality $\langle\cdot\rangle=\operatorname{Proj}_{\text {ker } \mathcal{T}}$ implies

$$
\operatorname{ker}\langle\cdot\rangle=(\operatorname{ker} \mathcal{T})^{\perp}=\left(\operatorname{ker} \mathcal{T}^{\star}\right)^{\perp}=\overline{\text { Range } \mathcal{T}} .
$$

In particular Range $\mathcal{T} \subset \operatorname{ker}\langle\cdot\rangle$.

The next point is very important as it relates to the solvability of $\mathcal{T} u=v$. We prove that the range of $\mathcal{T}$ is closed and derive the corresponding Poincaré type inequality, provided the magnetic field remains away from 0 (cf. [5] Proposition 2.2).

Proposition 3.2 We assume that $\inf _{x \in \mathbb{R}^{2}} B(x)>0$. Then $\mathcal{T}$ restricted to $\operatorname{ker}\langle\cdot\rangle$ is one to one map onto $\operatorname{ker}\langle\cdot\rangle$. Its inverse belongs to $\mathcal{L}(\operatorname{ker}\langle\cdot\rangle, \operatorname{ker}\langle\cdot\rangle)$ and we have the Poincaré inequality

$$
\|u\| \leq \frac{2 \pi}{\left|\omega_{0}\right|}\|\mathcal{T} u\|, \quad \omega_{0}=\frac{q}{m} \inf _{x \in \mathbb{R}^{2}} B(x) \neq 0
$$

for any $u \in \mathrm{D}(\mathcal{T}) \cap \operatorname{ker}\langle\cdot\rangle$.

Another useful result is given by

Proposition 3.3 Assume that $\varphi$ is a $C^{1}\left(\mathbb{R}^{2} \times \mathbb{R}^{2}\right)$ function such that $\varphi$ and $\mathcal{T} \varphi$ are bounded on $\mathbb{R}^{2} \times \mathbb{R}^{2}$. Then, for any function $u \in \mathrm{D}(\mathcal{T})$ we have the integration by parts formula

$$
\langle\varphi \mathcal{T} u\rangle+\langle u \mathcal{T} \varphi\rangle=0 .
$$

Proof. We have $\varphi u \in \mathrm{D}(\mathcal{T})$ and $\mathcal{T}(\varphi u)=\varphi \mathcal{T} u+u \mathcal{T} \varphi$. Taking the average yields

$$
\langle\varphi \mathcal{T} u\rangle+\langle u \mathcal{T} \varphi\rangle=\langle\mathcal{T}(\varphi u)\rangle=0
$$

since Range $\mathcal{T} \subset \operatorname{ker}\langle\cdot\rangle$.

\subsection{Averaging and first order differential operators}

In the sequel we need to analyze the commutation properties of averaging and transport operators. For the sake of the presentation we perform our analysis in the general framework of characteristic flows associated to divergence free vector fields. We denote by $b^{0}$ a given smooth, divergence free vector field $b^{0}: \mathbb{R}^{m} \rightarrow \mathbb{R}^{m}$ whose characteristic flow $Y=Y(s ; y)$ is well defined

$$
\begin{cases}\frac{d}{d s} Y & =b^{0}(Y(s ; y)), \quad(s, y) \in \mathbb{R} \times \mathbb{R}^{m} \\ Y(0 ; y) & =y, \quad y \in \mathbb{R}^{m}\end{cases}
$$

We intend to apply the following general results to the gyroaverage context and therefore, without loss of generality, we will assume that the characteristic flow $Y=Y(s ; y)$ is periodic i.e., for any $y \in \mathbb{R}^{m}$ there is $T_{c}(y)>0$ such that

$$
Y\left(T_{c}(y) ; y\right)=y \text {. }
$$

We may assume that $T_{c}(y)$ is the smallest positive period, excepted at those points $y \in \mathbb{R}^{m}$ where $b^{0}(y)=0$ when $s \rightarrow Y(s ; y)$ is constant. The non periodic case is beyond the scope of this work. Nevertheless notice that it can be handled as well, the average operator associated to a non periodic flow being defined through ergodic mean (see [4] for details)

$$
\langle u\rangle(y)=\lim _{T \rightarrow+\infty} \frac{1}{T} \int_{0}^{T} u(Y(s ; y)) \mathrm{d} s, y \in \mathbb{R}^{m} .
$$

For the sake of clarity, the results formulated in the general framework are stated by using latin upper case $T, A, B, C, \ldots$ whereas their counterpart results, referring to the gyroaverage framework, are stated by using calligraphy upper case $\mathcal{T}, \mathcal{A}, \mathcal{B}, \mathcal{C}, \ldots$ 
Proposition 3.4 The map $y \rightarrow T_{c}(y)$ is constant along the flow $Y$.

Proof. Indeed, for any $y \in \mathbb{R}^{m}$ such that $b^{0}(y) \neq 0$ let us consider $y_{h}=Y(h ; y)$, with $h \in \mathbb{R}$. Clearly $b^{0}\left(y_{h}\right) \neq 0$ (otherwise $Y(\cdot ; y)=y$ and $b^{0}(y)=b^{0}\left(y_{h}\right)=0$ ) and

$$
Y\left(T_{c}(y) ; y_{h}\right)=Y\left(T_{c}(y)+h ; y\right)=Y\left(h ; Y\left(T_{c}(y) ; y\right)\right)=Y(h ; y)=y_{h},
$$

which implies $T_{c}\left(y_{h}\right) \leq T_{c}(y)$. Replacing now $y$ by $y_{-h}$ (which satisfies $b^{0}\left(y_{-h}\right) \neq 0$ ) we deduce that $T_{c}(y) \leq T_{c}\left(y_{-h}\right)$ for any $h \in \mathbb{R}$ and therefore, after changing $h$ by $-h$ in the last inequality we obtain $T_{c}\left(y_{h}\right) \leq T_{c}(y) \leq T_{c}\left(y_{h}\right)$, saying that $T_{c}(y)$ is constant along the flow $Y$.

Next, we denote by $T$ the linear operator defined by $T u=\operatorname{div}_{y}\left(u(y) b^{0}(y)\right)$ for any $u$ in the domain

$$
\mathrm{D}(T)=\left\{u \in L^{2}\left(\mathbb{R}^{m}\right): \operatorname{div}_{y}\left(b^{0}(y) u(y)\right) \in L^{2}\left(\mathbb{R}^{m}\right)\right\},
$$

and thus the kernel of $T$ is given by constant functions along the characteristic flow (22)

$$
\operatorname{ker} T=\left\{u \in L^{2}\left(\mathbb{R}^{m}\right): u(Y(s ; y))=u(y), s \in \mathbb{R}, \text { a.e. } y \in \mathbb{R}^{m}\right\} .
$$

For further computations it is very useful to pick appropriate coordinates. Since we are interested in averaging along a characteristic flow (and therefore since the functions left invariant along this flow play a crucial role) we take as new coordinates a complete family of invariants $\left\{\psi_{1}, \ldots, \psi_{m-1}\right\}$ for the field $b^{0}$, together with a parametrization $\psi_{0}$ along its flow. To any invariant $\psi_{i}, i \in\{1, \ldots, m-1\}$ we associate a derivation $b^{i} \cdot \nabla_{y}$ acting, with respect to the new coordinates, like the partial derivative $\partial_{\psi_{i}}$. Assume that the vector field $b^{0}$ has a complete family of smooth independent prime integrals denoted $\psi_{1}=\psi_{1}(y), \ldots, \psi_{m-1}=\psi_{m-1}(y)$ i.e.,

$$
b^{0} \cdot \nabla_{y} \psi_{i}=0, \quad i \in\{1, \ldots, m-1\}, \quad \operatorname{rank} \partial_{y}{ }^{t}\left(\psi_{1}, \ldots, \psi_{m-1}\right)=m-1, \quad y \in \mathbb{R}^{m} .
$$

Moreover, assume that there is a function $\psi_{0}=\psi_{0}(y)$, whose gradient $\nabla_{y} \psi_{0}$ is well defined a.e. $y \in \mathbb{R}^{m}$, such that

$$
b^{0} \cdot \nabla_{y} \psi_{0}=I(y) \neq 0, \text { a.e. } y \in \mathbb{R}^{m}
$$

for some function $I \in \operatorname{ker} T$. Notice that $\psi_{0}$, satisfying (26), cannot be continuous everywhere. In general, $\psi_{0}$ has a discontinuity point, say $y_{0}$, on each characteristic and the jump $\left[\psi_{0}\right]$ around the discontinuity is given by

$$
\lim _{s \nearrow T_{c}\left(y_{0}\right)} \psi_{0}\left(Y\left(s ; y_{0}\right)\right)-\lim _{s \searrow 0} \psi_{0}\left(Y\left(s ; y_{0}\right)\right)=\int_{0}^{T_{c}\left(y_{0}\right)}\left(b^{0} \cdot \nabla_{y} \psi_{0}\right)\left(Y\left(s ; y_{0}\right)\right) \mathrm{d} s=T_{c}\left(y_{0}\right) I\left(y_{0}\right) .
$$

That means $\psi_{0}(y)$ is an angular coordinate. Since $T_{c}(\cdot), I(\cdot) \in \operatorname{ker}\left(b^{0} \cdot \nabla_{y}\right)$ it is possible to normalize the jump. Indeed, taking $\tilde{\psi}_{0}=\psi_{0} /\left[\psi_{0}\right]$ leads to

$$
b^{0} \cdot \nabla_{y} \tilde{\psi}_{0}=\frac{b^{0} \cdot \nabla_{y} \psi_{0}}{\left[\psi_{0}\right]}=\frac{I(y)}{T_{c}(y) I(y)}=\frac{1}{T_{c}(y)} \in \operatorname{ker}\left(b^{0} \cdot \nabla_{y}\right), \quad\left[\tilde{\psi}_{0}\right]=1 .
$$

Therefore we assume, without loss of generality, that the angular coordinate $\psi_{0}$ has the same jump on every characteristic. The following result is standard. For the sake of the completeness we detail the proof in Appendix A.

Proposition 3.5 Assume that the vector field $b^{0}$ has a complete family of smooth independent prime integrals (25). Moreover we assume that there is an angular coordinate (26) with constant jump $S=T_{c} I$ on every characteristic. Then, the following two properties are satisfied 
1. The map $y \rightarrow\left(\psi_{0}(y), \psi_{1}(y), \ldots, \psi_{m-1}(y)\right)$ is a bijection between $\mathbb{R}^{m}$ and $[0, S) \times D$, where $D:=\left\{\left(\psi_{1}(y), \ldots, \psi_{m-1}(y)\right): y \in \mathbb{R}^{m}\right\}$.

2. For any $i \in\{1, \ldots, m-1\}$ there is a unique vector field $b^{i}$ such that

$$
b^{i} \cdot \nabla_{y} \psi_{j}=\delta_{j}^{i}, \quad j \in\{0,1, \ldots, m-1\} .
$$

Remark 3.2 Any function $u=u(y)$ writes $u(y)=w\left(\psi_{0}(y), \psi_{1}(y), \ldots, \psi_{m-1}(y)\right)$, with $w$ defined on $[0, S) \times D$. Notice that if $u$ is continuous then $\lim _{r} \nearrow_{S} w\left(r, \psi_{1}, \ldots, \psi_{m-1}\right)=w\left(0, \psi_{1}, \ldots, \psi_{m-1}\right)$. Indeed, for any $\left(\psi_{1}, \ldots, \psi_{m-1}\right) \in D$ let us denote by $y_{0}$ the discontinuity point of $\psi_{0}$ along the characteristic associated to the invariants $\left(\psi_{1}, \ldots, \psi_{m-1}\right)$. We have

$$
\begin{aligned}
w\left(0, \psi_{1}, \ldots, \psi_{m-1}\right) & =u\left(y_{0}\right)=\lim _{s \nearrow T_{c}\left(y_{0}\right)} u\left(Y\left(s ; y_{0}\right)\right) \\
& =\lim _{s \nearrow T_{c}\left(y_{0}\right)} w\left(\psi_{0}\left(Y\left(s ; y_{0}\right)\right), \psi_{1}\left(y_{0}\right), \ldots, \psi_{m-1}\left(y_{0}\right)\right) \\
& =\lim _{s \nearrow T_{c}\left(y_{0}\right)} w\left(\psi_{0}\left(y_{0}\right)+s I, \psi_{1}, \ldots, \psi_{m-1}\right) \\
& =\lim _{r \nearrow S} w\left(r, \psi_{1}, \ldots, \psi_{m-1}\right) .
\end{aligned}
$$

If $u$ is continuous, the function $w$ above extends by $S$-periodicity with respect to $\psi_{0}$ to a continuous function, still denoted $w$, defined on $\mathbb{R} \times D$.

Remark 3.3 In our case (7) the divergence free vector field to be considered is $b^{0}: \mathbb{R}^{4} \rightarrow \mathbb{R}^{4}$, $b^{0}(x, v)=\left(0,0, \omega_{c}(x)^{\perp} v\right)$. A complete family of prime integrals for this vector field is given by (12) and we pick as angular coordinate $\psi_{0}(x, v)=-\alpha(v)$ where $\alpha(v)$ is the angle of $v \in \mathbb{R}^{2} \backslash\{(0,0)\}$ i.e.,

$$
v_{1}=|v| \cos \alpha(v), \quad v_{2}=|v| \sin \alpha(v), \quad \alpha(v) \in[0,2 \pi) .
$$

The angular coordinate $\psi_{0}(x, v)$ is discontinuous on $\mathbb{R}_{+}^{*} \times\{0\}$. But its gradient, which is well defined for any $v \in \mathbb{R}^{2} \backslash\left(\mathbb{R}_{+} \times\{0\}\right)$, can be extended by continuity on $\mathbb{R}^{2} \backslash\{(0,0)\}$

$$
\nabla_{v} \alpha=-\frac{{ }^{\perp} v}{|v|^{2}}, \quad \nabla_{x, v} \psi_{0}=\left(0,0, \frac{{ }^{\perp} v}{|v|^{2}}\right)
$$

and we have $I=\omega_{c}(x)^{\perp} v \cdot \nabla_{v} \psi_{0}=\omega_{c}(x) \in \operatorname{ker} \mathcal{T}$. On each characteristic $\psi_{0}$ has a jump of $S=T_{c}(x) \omega_{c}(x)=2 \pi$.

In addition, the vector fields $\left(b^{i}\right)_{i \in\{1,2,3\}}$ associated to the invariants $\psi_{1}=x_{1}, \psi_{2}=$ $x_{2}, \psi_{3}=|v|$ and the angular coordinate $\psi_{0}=-\alpha(v)$ are given by

$$
b^{1}=(1,0,0,0), \quad b^{2}=(0,1,0,0), \quad b^{3}=\left(0,0, \frac{v}{|v|}\right) .
$$

The vector fields $\left(b^{i}\right)_{i \in\{1, \ldots, m-1\}}$ will play a major role in our analysis cf. [5]. We shall establish several general results which mainly concern the commutation properties between the average and divergence operators, the inversion of $T=b^{0} \cdot \nabla_{y}$ on zero average functions and the construction of some auxiliary vector fields we will need for our further analysis. In particular we will show that after averaging, a transport operator reduces to another transport operator and we explicitely compute its advection field components. The following eight propositions explain in detail how to achieve these goals. Observe that the derivations along the vector fields $\left(b^{i}\right)_{i \in\{1, \ldots, m-1\}}$ coincide with the partial derivatives with respect to the invariants $\left(\psi_{i}\right)_{i \in\{1, \ldots, m-1\}}$, see Appendix A for details. 
Proposition 3.6 Consider a function $u=u(y)$ and let us denote by $w=w\left(\psi_{0}, \ldots, \psi_{m-1}\right)$ : $\mathbb{R} \times D \rightarrow \mathbb{R}$ the $S$ periodic function with respect to $\psi_{0}$ such that $u(y)=w\left(\psi_{0}(y), \ldots, \psi_{m-1}(y)\right)$. If $u \in C^{1}\left(\mathbb{R}^{m}\right)$, then $w \in C^{1}(\mathbb{R} \times D)$ and satisfies the identities

$$
b^{0} \cdot \nabla_{y} u(y)=I(y) \partial_{\psi_{0}} w\left(\psi_{0}(y), \ldots, \psi_{m-1}(y)\right), \quad b^{i} \cdot \nabla_{y} u(y)=\partial_{\psi_{i}} w\left(\psi_{0}(y), \ldots, \psi_{m-1}(y)\right)
$$

for any $i \in\{1, \ldots, m-1\}$.

In the sequel, for any two smooth vector fields $b(y)$ and $c(y)$, the notation $[b, c]$ stands for the vector field $\left(b \cdot \nabla_{y}\right) c-\left(c \cdot \nabla_{y}\right) b$, which is the vector field of the transport operator $b \cdot \nabla_{y}\left(c \cdot \nabla_{y}\right)-c \cdot \nabla_{y}\left(b \cdot \nabla_{y}\right)$

$$
b \cdot \nabla_{y}\left(c \cdot \nabla_{y}\right)-c \cdot \nabla_{y}\left(b \cdot \nabla_{y}\right)=[b, c] \cdot \nabla_{y}
$$

The commutators between the fields $\left(b^{i}\right)_{i \in\{0, \ldots, m-1\}}$ come easily by Schwarz's theorem on mixed partial derivatives [28].

Proposition 3.7 With the previous notations we have

$$
\left[b^{i}, b^{0}\right]=\frac{b^{i} \cdot \nabla_{y} I}{I} b^{0}, \quad i \in\{0, \ldots, m-1\}
$$

and $\left[b^{i}, b^{j}\right]=0, i, j \in\{1, \ldots, m-1\}$, with $I=b^{0} \cdot \nabla_{y} \psi_{0}$.

Proof. The first statement is obvious when $i=0$. For any smooth function $u=u(y)$ and $i \in\{1, \ldots, m-1\}$ we have, after the change of coordinate $u(y)=w(\psi(y)), \psi=\left(\psi_{0}, \ldots, \psi_{m-1}\right)$

$$
\begin{aligned}
{\left[b^{i}, b^{0}\right] \cdot \nabla_{y} u } & =\left(b^{i} \cdot \nabla_{y}\right)\left(b^{0} \cdot \nabla_{y} u\right)-\left(b^{0} \cdot \nabla_{y}\right)\left(b^{i} \cdot \nabla_{y} u\right) \\
& =\left(b^{i} \cdot \nabla_{y}\right)\left(I \frac{\partial w}{\partial \psi_{0}} \circ \psi\right)-\left(b^{0} \cdot \nabla_{y}\right)\left(\frac{\partial w}{\partial \psi_{i}} \circ \psi\right) \\
& =\frac{b^{i} \cdot \nabla_{y} I}{I} b^{0} \cdot \nabla_{y} u .
\end{aligned}
$$

Similarly, for any $i, j \in\{1, \ldots, m-1\}$ we have

$$
\begin{aligned}
{\left[b^{i}, b^{j}\right] \cdot \nabla_{y} u } & =\left(b^{i} \cdot \nabla_{y}\right)\left(b^{j} \cdot \nabla_{y} u\right)-\left(b^{j} \cdot \nabla_{y}\right)\left(b^{i} \cdot \nabla_{y} u\right) \\
& =\left(\frac{\partial}{\partial \psi_{i}}\left(\frac{\partial w}{\partial \psi_{j}}\right)\right) \circ \psi-\left(\frac{\partial}{\partial \psi_{j}}\left(\frac{\partial w}{\partial \psi_{i}}\right)\right) \circ \psi=0 .
\end{aligned}
$$

Corollary 3.1 The operators $\left(b^{i} \cdot \nabla_{y}\right)_{i \in\{0, \ldots, m-1\}}$ leave invariant ker $b^{0} \cdot \nabla_{y}$.

Proof. By Proposition 3.7 we have for any $u \in \operatorname{ker}\left(b^{0} \cdot \nabla_{y}\right) \cap \mathrm{D}\left(b^{i} \cdot \nabla_{y}\right)$

$$
\left(b^{i} \cdot \nabla_{y}\right)\left(b^{0} \cdot \nabla_{y} u\right)-\left(b^{0} \cdot \nabla_{y}\right)\left(b^{i} \cdot \nabla_{y} u\right)=\frac{b^{i} \cdot \nabla_{y} I}{I}\left(b^{0} \cdot \nabla_{y} u\right)
$$

and therefore $\left(b^{0} \cdot \nabla_{y}\right)\left(b^{i} \cdot \nabla_{y} u\right)=0$.

We establish now some properties regarding the divergences of the fields $\left(b^{i}\right)_{i \in\{1, \ldots, m-1\}}$.

Proposition 3.8 The matrix $\left(b^{i} \cdot \nabla_{y}\left(\operatorname{div}_{y} b^{j}\right)\right)_{(i, j) \in\{0, \ldots, m-1\}}$ is symmetric. In particular, for any $i \in\{1, \ldots, m-1\}$ the divergence of $b^{i}$ is constant along the flow of $b^{0}$. 
Proof. By Proposition 3.7 we know that

$$
\left[b^{i}, b^{j}\right]=\lambda^{i j} b^{0}, \quad b^{0} \cdot \nabla_{y} \lambda^{i j}=0, \quad i, j \in\{0, \ldots, m-1\}
$$

since $I$ and $b^{i} \cdot \nabla_{y} I$ are constant along the flow of $b^{0}$ cf. Corollary 3.1. Therefore we have

$$
0=\operatorname{div}_{y}\left(\lambda^{i j} b^{0}\right)=\operatorname{div}_{y}\left[b^{i}, b^{j}\right]=\left(b^{i} \cdot \nabla_{y}\right)\left(\operatorname{div}_{y} b^{j}\right)-\left(b^{j} \cdot \nabla_{y}\right)\left(\operatorname{div}_{y} b^{i}\right) .
$$

In particular, taking $j=0$ and $i \in\{1, \ldots, m-1\}$ one gets $b^{0} \cdot \nabla_{y}\left(\operatorname{div}_{y} b^{i}\right)=0$, since $b^{0}$ is divergence free.

We investigate now the commutation properties between the operators $\left(b^{i} \cdot \nabla_{y}\right)_{i \in\{0, \ldots, m-1\}}$ and the average operator. Since the left hand side of the Boltzmann equation (7) can be written into conservation form, it is worth analyzing how to average the divergence of a vector field. We refer to [4] for the proof.

Proposition 3.9 With the previous notations, we have for any smooth vector field $\xi(y)=$ $\left(\xi_{1}(y), \ldots, \xi_{m}(y)\right)$

$$
\left\langle\operatorname{div}_{y} \xi\right\rangle=\operatorname{div}_{y}\left\{\sum_{i=1}^{m-1}\left\langle\xi \cdot \nabla_{y} \psi_{i}\right\rangle b^{i}\right\}=\operatorname{div}_{y}\left\{\frac{\left\langle\xi \cdot \nabla_{y} \psi_{0}\right\rangle}{b^{0} \cdot \nabla_{y} \psi_{0}} b^{0}+\sum_{i=1}^{m-1}\left\langle\xi \cdot \nabla_{y} \psi_{i}\right\rangle b^{i}\right\} .
$$

Corollary 3.2 Assume that the fields $\left(b^{i}\right)_{i \in\{1, \ldots, m-1\}}$ are smooth and have bounded divergence. Then the operators $\left(b^{i} \cdot \nabla_{y}\right)_{i \in\{0, \ldots, m-1\}}$ are commuting with the average operator.

Proof. Obviously $b^{0} \cdot \nabla_{y}$ and $\langle\cdot\rangle$ are commuting, since for any function $u \in \mathrm{D}\left(b^{0} \cdot \nabla_{y}\right)$ we have

$$
\left\langle b^{0} \cdot \nabla_{y} u\right\rangle=b^{0} \cdot \nabla_{y}\langle u\rangle=0 .
$$

For any $i \in\{1, \ldots, m-1\}$ and function $u \in \mathrm{D}\left(b^{i} \cdot \nabla_{y}\right)$ we obtain thanks to Proposition 3.9 applied to the field $u b^{i}$

$$
\left\langle\operatorname{div}_{y}\left(u b^{i}\right)\right\rangle=\operatorname{div}_{y}\left(\langle u\rangle b^{i}\right)
$$

and our conclusion follows easily, since we know that $\operatorname{div}_{y} b^{i} \in \operatorname{ker}\left(b^{0} \cdot \nabla_{y}\right)$.

Remark 3.4 In the particular case of the vector fields $b^{0}=\left(0,0, \omega_{c}(x){ }^{\perp} v\right), b^{1}(1,0,0,0)$, $b^{2}=(0,1,0,0), b^{3}=(0,0, v /|v|)$ we obtain for any smooth vector field $\xi=\left(\xi_{x}(x, v), \xi_{v}(x, v)\right)$ the formulae, thanks to the identity $\operatorname{div}_{v}\left\{v /|v|^{2}\right\}=0$

$$
\left\langle\operatorname{div}_{x, v} \xi\right\rangle=\operatorname{div}_{x}\left\langle\xi_{x}\right\rangle+\frac{v}{|v|^{2}} \cdot \nabla_{v}\left\langle\xi_{v} \cdot v\right\rangle,\left\langle\operatorname{div}_{x} \xi_{x}\right\rangle=\left\langle\operatorname{div}_{x, v}\left(\xi_{x}, 0\right)\right\rangle=\operatorname{div}_{x}\left\langle\xi_{x}\right\rangle .
$$

The previous result guarantees that a transport operator reduces, after average, to another transport operator and allows us to keep the model into conservative form cf. [5]. For the sake of transparency we detail this computation in the following proposition.

Proposition 3.10 Consider $a=a(y)$ a smooth divergence free vector field in $\mathbb{R}^{m}$. Then there is a divergence free vector field $A=A(y)$ such that for any smooth function $u \in$ $\operatorname{ker}\left(b^{0} \cdot \nabla_{y}\right) \cup\left\{\psi_{0}\right\}$

$$
\left\langle\operatorname{div}_{y}(u a)\right\rangle=\operatorname{div}_{y}(u A) .
$$

Moreover, such a vector field is unique and given by

$$
A=\frac{\left\langle a \cdot \nabla_{y} \psi_{0}\right\rangle}{I} b^{0}+\sum_{i=1}^{m-1}\left\langle a \cdot \nabla_{y} \psi_{i}\right\rangle b^{i} .
$$


Proof. Apply Proposition 3.9 to the field $y \rightarrow u(y) a(y)$, for any $u \in \operatorname{ker}\left(b^{0} \cdot \nabla_{y}\right)$

$$
\begin{aligned}
\left\langle\operatorname{div}_{y}(u a)\right\rangle & =\operatorname{div}_{y}\left\{\left\langle u a \cdot \nabla_{y} \psi_{0}\right\rangle b^{0} I^{-1}+\sum_{i=1}^{m-1}\left\langle u a \cdot \nabla_{y} \psi_{i}\right\rangle b^{i}\right\} \\
& =\operatorname{div}_{y}\left\{u\left\langle a \cdot \nabla_{y} \psi_{0}\right\rangle b^{0} I^{-1}+\sum_{i=1}^{m-1} u\left\langle a \cdot \nabla_{y} \psi_{i}\right\rangle b^{i}\right\} .
\end{aligned}
$$

In particular it is easily seen, that setting $u=1$ and defining $A=\left\langle a \cdot \nabla_{y} \psi_{0}\right\rangle b^{0} I^{-1}+$ $\sum_{i=1}^{m-1}\left\langle a \cdot \nabla_{y} \psi_{i}\right\rangle b^{i}$, the field $A$ is unique and divergence free.

Remark 3.5 If $\left(\alpha_{0}, \ldots, \alpha_{m-1}\right)$ are the coordinates of a with respect to the basis $\left\{b^{0}, \ldots, b^{m-1}\right\}$, then $\left(\left\langle\alpha_{0}\right\rangle, \ldots,\left\langle\alpha_{m-1}\right\rangle\right)$ are the coordinates of $A$ with respect to the same basis

$$
a=\sum_{i=0}^{m-1} \alpha_{i} b^{i}, \quad A=\sum_{i=0}^{m-1}\left\langle\alpha_{i}\right\rangle b^{i} .
$$

Remark 3.6 In the particular case of the vector fields $b^{0}=\left(0,0, \omega_{c}(x){ }^{\perp} v\right), b^{1}(1,0,0,0)$, $b^{2}=(0,1,0,0), b^{3}=(0,0, v /|v|), a=(v, q / m E)$ we obtain the coefficients

$$
\alpha_{0}=\frac{q E}{m \omega_{c}(x)} \cdot \frac{\perp_{v}}{|v|^{2}}, \quad \alpha_{1}=v_{1}, \quad \alpha_{2}=v_{2}, \quad \alpha_{3}=\frac{q E}{m} \cdot \frac{v}{|v|} .
$$

It is easily seen that $\left\langle\alpha_{i}\right\rangle=0, i \in\{0, \ldots, 3\}$ and thus $\mathcal{A}=\sum_{i=0}^{3}\left\langle\alpha_{i}\right\rangle b^{i}=0$.

Notice that for any $i \in\{0, \ldots, m-1\}$ the function $\alpha_{i}-\left\langle\alpha_{i}\right\rangle$ has zero average, and thus, by Proposition 3.2, there is a unique $\beta_{i}$ satisfying $b^{0} \cdot \nabla_{y} \beta_{i}=\alpha_{i}-\left\langle\alpha_{i}\right\rangle$, with $\left\langle\beta_{i}\right\rangle=0$. Therefore, we denote by $B$ the vector field

$$
B=\sum_{i=0}^{m-1} \beta_{i} b^{i}
$$

whose characterization makes the object of the next proposition.

Proposition 3.11 Consider $a=a(y)$ a smooth, divergence free vector field in $\mathbb{R}^{m}$. Let $A$ and $B$ be the vector fields defined in (29) and (31) respectively.

i) Then for any smooth function $u \in \operatorname{ker}\left(b^{0} \cdot \nabla_{y}\right) \cup\left\{\psi_{0}\right\}$ we have

$$
\operatorname{div}_{y}\left\{\left(B \cdot \nabla_{y} u\right) b^{0}\right\}=\operatorname{div}_{y}\{u(a-A)\}
$$

and $\operatorname{div}_{y}(I B)=0$.

ii) Let $\tilde{B}=B+\lambda_{0} b^{0}$ where $b^{0} \cdot \nabla_{y} \lambda_{0}=\frac{B \cdot \nabla_{y} I}{I},\left\langle\lambda_{0}\right\rangle=0$. Then for any smooth function $u \in \operatorname{ker}\left(b^{0} \cdot \nabla_{y}\right)$ we have

$$
\operatorname{div}_{y}\left\{\left(\tilde{B} \cdot \nabla_{y} u\right) b^{0}\right\}=\operatorname{div}_{y}\{u(a-A)\}, \operatorname{div}_{y} \tilde{B}=0 .
$$

Proof. i) For any smooth function $u \in \operatorname{ker}\left(b^{0} \cdot \nabla_{y}\right)$, taking into account that $\left(b^{i} \cdot \nabla_{y}\right)_{i \in\{0, \ldots, m-1\}}$ leave invariant $\operatorname{ker}\left(b^{0} \cdot \nabla_{y}\right)$, we have

$$
\operatorname{div}_{y}\left\{\left(B \cdot \nabla_{y} u\right) b^{0}\right\}=b^{0} \cdot \nabla_{y}\left(B \cdot \nabla_{y} u\right)=\sum_{i=0}^{m-1}\left(b^{i} \cdot \nabla_{y} u\right)\left(b^{0} \cdot \nabla_{y} \beta_{i}\right)=\operatorname{div}_{y}\{u(a-A)\}
$$


Taking now $u=\psi_{0}$ one gets

$$
b^{0} \cdot \nabla_{y}\left(B \cdot \nabla_{y} \psi_{0}\right)=b^{0} \cdot \nabla_{y}\left(\beta_{0} I\right)=I b^{0} \cdot \nabla_{y} \beta_{0}=I\left(\alpha_{0}-\left\langle\alpha_{0}\right\rangle\right)=(a-A) \cdot \nabla_{y} \psi_{0} .
$$

It remains to establish that $I B$ is divergence free. Notice that for any smooth function $u \in \operatorname{ker}\left(b^{0} \cdot \nabla_{y}\right)$, the equation (32) becomes

$$
\left[b^{0}, B\right] \cdot \nabla_{y} u=b^{0} \cdot \nabla_{y}\left(B \cdot \nabla_{y} u\right)-B \cdot \nabla_{y}\left(b^{0} \cdot \nabla_{y} u\right)=(a-A) \cdot \nabla_{y} u .
$$

In particular taking $u \in\left\{\psi_{1}, \ldots, \psi_{m-1}\right\}$ we deduce that

$$
\left\{\left[B, b^{0}\right]+a-A\right\} \perp \operatorname{span}\left\{\nabla_{y} \psi_{1}, \ldots, \nabla_{y} \psi_{m-1}\right\}=\left(\mathbb{R} b^{0}\right)^{\perp}
$$

implying that

$$
\left[B, b^{0}\right]+a-A=\lambda b^{0}
$$

Taking now $u=\psi_{0}$ we obtain

$$
B \cdot \nabla_{y}\left(b^{0} \cdot \nabla_{y} \psi_{0}\right)-b^{0} \cdot \nabla_{y}\left(B \cdot \nabla_{y} \psi_{0}\right)+(a-A) \cdot \nabla_{y} \psi_{0}=\lambda I
$$

and since we know that $b^{0} \cdot \nabla_{y}\left(B \cdot \nabla_{y} \psi_{0}\right)=(a-A) \cdot \nabla_{y} \psi_{0}$ we deduce that

$$
B \cdot \nabla_{y} I=\lambda I .
$$

Taking the divergence of (34) implies $-b^{0} \cdot \nabla_{y} \operatorname{div}_{y} B=b^{0} \cdot \nabla_{y} \lambda$ and therefore

$$
\operatorname{div}_{y} B+\lambda=\mu \in \operatorname{ker}\left(b^{0} \cdot \nabla_{y}\right) .
$$

Combining (35), (36) yields $\operatorname{div}_{y}(I B)=\mu I, b^{0} \cdot \nabla_{y} \mu=0$. We are done if we prove that $\operatorname{div}_{y}(I B)$ has zero average, since, in this case we write

$$
\mu I=\langle\mu I\rangle=\left\langle\operatorname{div}_{y}(I B)\right\rangle=0 .
$$

Indeed, by Proposition 3.9 we have

$$
\left\langle\operatorname{div}_{y}(I B)\right\rangle=\operatorname{div}_{y}\left\{\sum_{i=1}^{m-1}\left\langle I B \cdot \nabla_{y} \psi_{i}\right\rangle b^{i}\right\}=\operatorname{div}_{y}\left\{\sum_{i=1}^{m-1}\left\langle I \beta_{i}\right\rangle b^{i}\right\}=0 .
$$

ii) Obviously we have $\operatorname{div}_{y} \tilde{B}=\operatorname{div}_{y} B+b^{0} \cdot \nabla_{y} \lambda_{0}=\operatorname{div}_{y}(I B) / I=0$ and for any smooth function $u \in \operatorname{ker}\left(b^{0} \cdot \nabla_{y}\right)$ we can write

$$
\operatorname{div}_{y}\left\{\left(\tilde{B} \cdot \nabla_{y} u\right) b^{0}\right\}=\operatorname{div}_{y}\left\{\left(B \cdot \nabla_{y} u\right) b^{0}\right\}=\operatorname{div}_{y}\{u(a-A)\}
$$

Remark 3.7 For any smooth function $u \in \operatorname{ker}\left(b^{0} \cdot \nabla_{y}\right) \cup\left\{\psi_{0}\right\}$ we have $\left\langle B \cdot \nabla_{y} u\right\rangle=\left\langle\tilde{B} \cdot \nabla_{y} u\right\rangle=$ 0 .

Remark 3.8 For any function $u \in \operatorname{ker}\left(b^{0} \cdot \nabla_{y}\right)$ we have by Proposition 3.10

$$
\left\langle(a-A) \cdot \nabla_{y} u\right\rangle=\left\langle\operatorname{div}_{y}(u a)\right\rangle-\left\langle\operatorname{div}_{y}(u A)\right\rangle=0
$$

and therefore, by Proposition 3.2, we know that there is a unique zero average function $w$ solving $b^{0} \cdot \nabla_{y} w=(a-A) \cdot \nabla_{y} u$. Proposition 3.11 says that $w=\tilde{B} \cdot \nabla_{y} u=B \cdot \nabla_{y} u$. 
Remark 3.9 In our particular case (see Remark 3.6) we obtain the coefficients

$$
\beta_{0}=\frac{q E}{m \omega_{c}^{2}} \cdot \frac{v}{|v|^{2}}, \quad \beta_{1}=-\frac{v_{2}}{\omega_{c}}, \quad \beta_{2}=\frac{v_{1}}{\omega_{c}}, \quad \beta_{3}=-\frac{q E}{m \omega_{c}} \cdot \frac{{ }^{\perp} v}{|v|}=v_{\wedge} \cdot \frac{v}{|v|}
$$

where $v_{\wedge}={ }^{\perp} E / B$ is the electric cross field drift, and therefore

$$
\begin{aligned}
& \mathcal{B}=\sum_{i=0}^{3} \beta_{i} b^{i}=\left(-\frac{{ }^{\perp} v}{\omega_{c}}, v_{\wedge}\right), \operatorname{div}_{x, v} \mathcal{B}=\frac{{ } v \cdot \nabla_{x} \omega_{c}}{\omega_{c}^{2}} \\
& \lambda_{0}=-\frac{v \cdot \nabla_{x} \omega_{c}}{\omega_{c}^{3}}, \tilde{\mathcal{B}}=\left(-\frac{{ }^{\perp} v}{\omega_{c}}, v_{\wedge}-\frac{v \cdot \nabla_{x} \omega_{c}}{\omega_{c}^{2}} \perp v\right) .
\end{aligned}
$$

For any function $u(x, v) \in \operatorname{ker} \mathcal{T}$ the unique zero average function $w(x, v)$ solving

$$
\mathcal{T} w=(a-\mathcal{A}) \cdot \nabla_{x, v} u=a \cdot \nabla_{x, v} u=v \cdot \nabla_{x} u+\frac{q}{m} E \cdot \nabla_{v} u
$$

is given by

$$
w=\tilde{\mathcal{B}} \cdot \nabla_{x, v} u=\mathcal{B} \cdot \nabla_{x, v} u=-\frac{{ }^{\perp} v}{\omega_{c}(x)} \cdot \nabla_{x} u+v_{\wedge} \cdot \nabla_{v} u
$$

The last field we compute will be useful when deriving the second order approximation, in Section 4.2 .

Proposition 3.12 Consider $a=a(y)$ a smooth, divergence free vector field in $\mathbb{R}^{m}$ and let $\tilde{B}$ be the vector field defined in Proposition 3.11, ii). Then there is a divergence free vector field $C=C(y)$ such that for any smooth function $u \in \operatorname{ker}\left(b^{0} \cdot \nabla_{y}\right) \cup\left\{\psi_{0}\right\}$ we have

$$
\left\langle\operatorname{div}_{y}\left\{\left(\tilde{B} \cdot \nabla_{y} u\right) a\right\}\right\rangle=\operatorname{div}_{y}(u C) .
$$

Proof. For the field $B$ given by (31) and for any smooth function $u \in \operatorname{ker}\left(b^{0} \cdot \nabla_{y}\right)$ we have by Proposition 3.9

$$
\begin{aligned}
\left\langle\operatorname{div}_{y}\left\{\left(\tilde{B} \cdot \nabla_{y} u\right) a\right\}\right\rangle & =\operatorname{div}_{y}\left\{\sum_{i=1}^{m-1}\left\langle\left(B \cdot \nabla_{y} u\right)\left(a \cdot \nabla_{y} \psi_{i}\right)\right\rangle b^{i}\right\} \\
& =\operatorname{div}_{y}\left\{\sum_{i=1}^{m-1} \sum_{j=1}^{m-1}\left\langle\alpha_{i} \beta_{j}\right\rangle\left(b^{j} \cdot \nabla_{y} u\right) b^{i}\right\} \\
& =\sum_{j=1}^{m-1} \operatorname{div}_{y}\left\{\sum_{i=1}^{m-1}\left\langle\alpha_{i} \beta_{j}\right\rangle b^{i}\right\}\left(b^{j} \cdot \nabla_{y} u\right) \\
& +\sum_{j=1}^{m-1} \sum_{i=1}^{m-1}\left\langle\alpha_{i} \beta_{j}\right\rangle b^{i} \cdot \nabla_{y}\left(b^{j} \cdot \nabla_{y} u\right) .
\end{aligned}
$$

The last term vanishes, as a contraction between the anti-symmetric matrix $\left(\left\langle\alpha_{i} \beta_{j}\right\rangle\right)_{i, j}$ and the symmetric matrix $\left(b^{i} \cdot \nabla_{y}\left(b^{j} \cdot \nabla_{y} u\right)\right)_{i, j}$. Indeed, by Proposition 3.3 we obtain

$$
\left\langle\alpha_{i} \beta_{j}\right\rangle=\left\langle\left(\alpha_{i}-\left\langle\alpha_{i}\right\rangle\right) \beta_{j}\right\rangle=\left\langle\left(b^{0} \cdot \nabla_{y} \beta_{i}\right) \beta_{j}\right\rangle=-\left\langle\beta_{i}\left(b^{0} \cdot \nabla_{y} \beta_{j}\right)\right\rangle=-\left\langle\alpha_{j} \beta_{i}\right\rangle
$$

and by Proposition 3.7 one gets

$$
b^{i} \cdot \nabla_{y}\left(b^{j} \cdot \nabla_{y} u\right)-b^{j} \cdot \nabla_{y}\left(b^{i} \cdot \nabla_{y} u\right)=\left[b^{i}, b^{j}\right] \cdot \nabla_{y} u=0, \quad i, j \in\{1, \ldots, m-1\} .
$$


Using again Proposition 3.9, we deduce that for any $j \in\{1, \ldots, m-1\}$

$$
\operatorname{div}_{y}\left\{\sum_{i=1}^{m-1}\left\langle\alpha_{i} \beta_{j}\right\rangle b^{i}\right\}=\operatorname{div}_{y}\left\{\sum_{i=1}^{m-1}\left\langle\beta_{j} a \cdot \nabla_{y} \psi_{i}\right\rangle b^{i}\right\}=\left\langle\operatorname{div}_{y}\left(\beta_{j} a\right)\right\rangle .
$$

Combining (37), (38) we obtain

$$
\left\langle\operatorname{div}_{y}\left\{\left(\tilde{B} \cdot \nabla_{y} u\right) a\right\}\right\rangle=\sum_{j=1}^{m-1} \gamma_{j} b^{j} \cdot \nabla_{y} u=\sum_{j=0}^{m-1} \gamma_{j} b^{j} \cdot \nabla_{y} u
$$

with the notation

$$
\gamma_{0}=\frac{\left.\left\langle\operatorname{div}_{y}\left(\beta_{0}+\lambda_{0}\right) I a\right)\right\rangle}{I}, \quad \gamma_{j}=\left\langle\operatorname{div}_{y}\left(\beta_{j} a\right)\right\rangle, \quad j \in\{1, \ldots, m-1\}, \quad C=\sum_{j=0}^{m-1} \gamma_{j} b^{j} .
$$

It is easily seen that the above formula still holds true for $u=\psi_{0}$. It remains to prove that $\operatorname{div}_{y} C=0$. Since $\operatorname{div}_{y}\left(\gamma_{0} b^{0}\right)=0$, it is enough to prove that $\operatorname{div}_{y}\left\{\sum_{i=1}^{m-1} \gamma_{i} b^{i}\right\}=0$. For any smooth function $u \in \operatorname{ker}\left(b^{0} \cdot \nabla_{y}\right)$ we have

$$
-\int_{\mathbb{R}^{m}} u \operatorname{div}_{y} C \mathrm{~d} y=\int_{\mathbb{R}^{m}} C \cdot \nabla_{y} u \mathrm{~d} y=\int_{\mathbb{R}^{m}}\left\langle\operatorname{div}_{y}\left\{\left(B \cdot \nabla_{y} u\right) a\right\}\right\rangle \mathrm{d} y=\int_{\mathbb{R}^{m}} \operatorname{div}_{y}\left\{\left(B \cdot \nabla_{y} u\right) a\right\} \mathrm{d} y=0
$$

and therefore $\left\langle\operatorname{div}_{y} C\right\rangle=0$. But $\operatorname{div}_{y} C$ is constant along the flow of $b^{0}$

$$
\operatorname{div}_{y} C=\sum_{i=1}^{m-1} \gamma_{i} \operatorname{div}_{y} b^{i}+\sum_{i=1}^{m-1} b^{i} \cdot \nabla_{y} \gamma_{i}
$$

since $\gamma_{i}, \operatorname{div}_{y} b^{i}, b^{i} \cdot \nabla_{y} \gamma_{i}$ are constant along the same flow. Therefore $\operatorname{div}_{y} C=\left\langle\operatorname{div}_{y} C\right\rangle=0$.

Remark 3.10 Actually we will need a divergence free vector field $C$ satisfying the statement in Proposition 3.12 only for functions $u \in \operatorname{ker}\left(b^{0} \cdot \nabla_{y}\right)$. The coordinates along $\left(b^{i}\right)_{i \in\{1, \ldots, m-1\}}$ of such vector fields are uniquely determined by $\gamma_{i}=\left\langle\operatorname{div}_{y}\left(\beta_{i} a\right)\right\rangle, i \in\{1, \ldots, m-1\}$ and the coordinate along $b^{0}$ could be any smooth function in $\operatorname{ker}\left(b^{0} \cdot \nabla_{y}\right)$, which guarantees the constraint $\operatorname{div}_{y} C=0$.

Remark 3.11 In the particular case of the vector fields $b^{0}=\left(0,0, \omega_{c}(x){ }^{\perp} v\right), b^{1}(1,0,0,0)$, $b^{2}=(0,1,0,0), b^{3}=(0,0, v /|v|), a=(v, q / m E)$ we obtain the coefficients

$$
\left(\gamma_{1}, \gamma_{2}\right)=-v_{\wedge}-v_{\mathrm{GD}}, \quad \gamma_{3}=-\frac{|v| v_{\wedge}}{2} \cdot \frac{\nabla_{x} B}{B}
$$

where $v_{\wedge}, v_{\mathrm{GD}}$ are the (rescaled) electric cross field drift and magnetic gradient drift

$$
v_{\wedge}=\frac{{ }^{\perp} E}{B}, \quad v_{\mathrm{GD}}=-\frac{|v|^{2}}{2 \omega_{c}(x)} \frac{{ }^{\perp} \nabla_{x} B}{B}
$$

and the vector field $\mathcal{C}$ becomes

$$
\mathcal{C}=\left(-v_{\wedge}-v_{\mathrm{GD}},-\frac{v_{\wedge} \cdot \nabla_{x} B}{2 B} v+\gamma_{0} \omega_{c}(x)^{{ }^{\perp}} v\right)
$$

for some $\gamma_{0} \in \operatorname{ker} \mathcal{T}$. It is easily seen that $\operatorname{div}_{x} v_{\wedge}=-\left(v_{\wedge} \cdot \nabla_{x} B\right) / B, \operatorname{div}_{x} v_{\mathrm{GD}}=0$ and thus $\operatorname{div}_{x, v} \mathcal{C}=0$. In the sequel we will consider $\gamma_{0}=0$. 
Proposition 3.13 Consider $a=a(y)$ a smooth, divergence free vector field in $\mathbb{R}^{m}$. Then for any smooth function $u$ we have

$$
\operatorname{div}_{y}\left\{\left(\tilde{B} \cdot \nabla_{y} u\right) A\right\}=\operatorname{div}_{y}\{u[A, \tilde{B}]\}+\operatorname{div}_{y}\left\{\left(A \cdot \nabla_{y} u\right) \tilde{B}\right\}
$$

where the vector fields $A$ and $\tilde{B}$ are given by (29) and Proposition 3.11, ii), respectively.

Proof. The field $[A, \tilde{B}]$ is divergence free

$$
\operatorname{div}_{y}[A, \tilde{B}]=A \cdot \nabla_{y} \operatorname{div}_{y} \tilde{B}-\tilde{B} \cdot \nabla_{y} \operatorname{div}_{y} A=0
$$

and therefore

$$
\begin{aligned}
\operatorname{div}_{y}\left\{\left(\tilde{B} \cdot \nabla_{y} u\right) A\right\} & =A \cdot \nabla_{y}\left(\tilde{B} \cdot \nabla_{y} u\right) \\
& =[A, \tilde{B}] \cdot \nabla_{y} u+\tilde{B} \cdot \nabla_{y}\left(A \cdot \nabla_{y} u\right) \\
& =\operatorname{div}_{y}\{u[A, \tilde{B}]\}+\operatorname{div}_{y}\left\{\left(A \cdot \nabla_{y} u\right) \tilde{B}\right\}
\end{aligned}
$$

Remark 3.12 Let $u \in \operatorname{ker}\left(b^{0} \cdot \nabla_{y}\right)$ and $z$ satisfying

$$
\operatorname{div}_{y}\{u(a-A)\}+\operatorname{div}_{y}\left\{z b^{0}\right\}=0,\langle z\rangle=0
$$

which means that $z=-\tilde{B} \cdot \nabla_{y} u$, cf. Proposition 3.11. Then combining Propositions 3.12 and 3.13 we obtain

$$
\begin{aligned}
\left\langle\operatorname{div}_{y}(z a)\right\rangle-\operatorname{div}_{y}(z A) & =-\left\langle\operatorname{div}_{y}\left\{\left(\tilde{B} \cdot \nabla_{y} u\right) a\right\}\right\rangle+\operatorname{div}_{y}\left\{\left(\tilde{B} \cdot \nabla_{y} u\right) A\right\} \\
& =-\operatorname{div}_{y}(u C)+\operatorname{div}_{y}\{u[A, \tilde{B}]\}+\operatorname{div}_{y}\left\{\left(A \cdot \nabla_{y} u\right) \tilde{B}\right\} \\
& =\operatorname{div}_{y}\left\{u([A, \tilde{B}]-C)+\left(A \cdot \nabla_{y} u\right) \tilde{B}\right\} .
\end{aligned}
$$

\subsection{Average and the collision operator}

In this section we will show that the average operator associated to $\mathcal{T}=\omega_{c}(x)^{\perp} v \cdot \nabla_{v}$ and the collision operator are commuting. This means that at the lowest order the collision mechanism is not affected by strong magnetic fields. Corrections appear only at the next orders, as commutators between transport and convolution through the scattering cross section, see Proposition 3.15. We first recall the classical properties of the linear Boltzmann operator $Q$. The notation $L_{M}^{2}$ stands for $L^{2}\left(\frac{\mathrm{d} v \mathrm{~d} x}{M(v)}\right)$.

Proposition 3.14 Assume that the scattering cross section satisfies (5). Then the gain/loss collision operators $Q_{ \pm}$

$$
Q_{+}(f)(x, v)=\frac{1}{\tau} \int_{\mathbb{R}^{2}} s\left(v, v^{\prime}\right) M(v) f\left(x, v^{\prime}\right) \mathrm{d} v^{\prime}, Q_{-}(f)(x, v)=\frac{1}{\tau} \int_{\mathbb{R}^{2}} s\left(v, v^{\prime}\right) M\left(v^{\prime}\right) f(x, v) \mathrm{d} v^{\prime}
$$

are linear bounded operators from $L_{M}^{2}$ to $L_{M}^{2}$ and $\left\|Q_{ \pm}\right\| \leq S_{0} / \tau$. Moreover, if the scattering cross section also satisfies (4), then $Q_{ \pm}$are symmetric with respect to the scalar product of $L_{M}^{2}$. 
Notice that $M$ depends only on $|v|$ and therefore $M \in \operatorname{ker} \mathcal{T}, \mathcal{T}=\omega_{c}(x){ }^{\perp} v \cdot \nabla_{v}$. Observe that the average operator is also bounded from $L_{M}^{2}$ to $L_{M}^{2}$. Indeed, for any $f \in L_{M}^{2}$ we have $f / \sqrt{M} \in L^{2}(\mathrm{~d} v \mathrm{~d} x)$ and thus

$$
\|\langle f\rangle\|_{L_{M}^{2}}^{2}=\left\|\left\langle\frac{f}{\sqrt{M}}\right\rangle\right\|_{L^{2}}^{2} \leq\left\|\frac{f}{\sqrt{M}}\right\|_{L^{2}}^{2}=\|f\|_{L_{M}^{2}}^{2} .
$$

We assume that there is a function $\sigma: \mathbb{R}_{+} \rightarrow\left[s_{0}, S_{0}\right]$ such that

$$
s\left(v, v^{\prime}\right)=\sigma\left(\left|v-v^{\prime}\right|\right), \quad v, v^{\prime} \in \mathbb{R} .
$$

Lemma 3.1 Assume that the scattering cross section satisfies (40). Then the gain/loss collision operators are commuting with the characteristic flow of $\mathcal{T}$. In particular the gain/loss collision operators leave invariant $L_{M}^{2} \cap \operatorname{ker} \mathcal{T}$ and $L_{M}^{2} \cap \operatorname{ker}\langle\cdot\rangle$.

Proof. Consider $f \in L_{M}^{2}$ and for any $\alpha \in \mathbb{R}$ let us introduce $f_{\alpha}(x, v)=f\left(x, R_{\alpha} v\right)$, where $R(\alpha)$ stands for the rotation of angle $\alpha$ (see (10)). Obviously $f_{\alpha} \in L_{M}^{2}$ and

$$
\begin{aligned}
\left(Q_{+} f_{\alpha}\right)(x, v) & =\frac{1}{\tau} \int_{\mathbb{R}^{2}} \sigma\left(\left|v-v^{\prime}\right|\right) M(v) f_{\alpha}\left(x, v^{\prime}\right) \mathrm{d} v^{\prime} \\
& =\frac{1}{\tau} \int_{\mathbb{R}^{2}} \sigma\left(\left|v-v^{\prime}\right|\right) M(v) f\left(x, R_{\alpha} v^{\prime}\right) \mathrm{d} v^{\prime} \\
& =\frac{1}{\tau} \int_{\mathbb{R}^{2}} \sigma\left(\left|v-R_{-\alpha} w^{\prime}\right|\right) M(v) f\left(x, w^{\prime}\right) \mathrm{d} w^{\prime} \\
& =\frac{1}{\tau} \int_{\mathbb{R}^{2}} \sigma\left(\left|R_{\alpha} v-w^{\prime}\right|\right) M\left(R_{\alpha} v\right) f\left(x, w^{\prime}\right) \mathrm{d} w^{\prime} \\
& =\left(Q_{+} f\right)\left(x, R_{\alpha} v\right) .
\end{aligned}
$$

Similarly one gets

$$
\begin{aligned}
\left(Q_{-} f_{\alpha}\right)(x, v) & =\frac{1}{\tau} \int_{\mathbb{R}^{2}} \sigma\left(\left|v-v^{\prime}\right|\right) M\left(v^{\prime}\right) f_{\alpha}(x, v) \mathrm{d} v^{\prime} \\
& =\frac{1}{\tau} \int_{\mathbb{R}^{2}} \sigma\left(\left|v-v^{\prime}\right|\right) M\left(v^{\prime}\right) f\left(x, R_{\alpha} v\right) \mathrm{d} v^{\prime} \\
& =\frac{1}{\tau} \int_{\mathbb{R}^{2}} \sigma\left(\left|R_{\alpha} v-R_{\alpha} v^{\prime}\right|\right) M\left(R_{\alpha} v^{\prime}\right) f\left(x, R_{\alpha} v\right) \mathrm{d} v^{\prime} \\
& =\frac{1}{\tau} \int_{\mathbb{R}^{2}} \sigma\left(\left|R_{\alpha} v-w^{\prime}\right|\right) M\left(w^{\prime}\right) f\left(x, R_{\alpha} v\right) \mathrm{d} w^{\prime} \\
& =\left(Q_{-} f\right)\left(x, R_{\alpha} v\right) .
\end{aligned}
$$

In particular, if $f \in L_{M}^{2} \cap \operatorname{ker} \mathcal{T}$ then

$$
\left(Q_{ \pm} f\right)\left(x, R_{\alpha} v\right)=\left(Q_{ \pm} f_{\alpha}\right)(x, v)=\left(Q_{ \pm} f\right)(x, v)
$$

saying that $Q_{ \pm} f \in \operatorname{ker} \mathcal{T}$. Consider now $h \in L_{M}^{2} \cap \operatorname{ker}\langle\cdot\rangle$ and $\varphi \in \operatorname{ker} \mathcal{T}$. Then $Q_{ \pm}(h) / \sqrt{M} \in$ $L^{2}(\mathrm{~d} v \mathrm{~d} x), \sqrt{M} \varphi \in L_{M}^{2}$ and we can write

$$
\int_{\mathbb{R}^{4}} \frac{Q_{ \pm}(h)}{\sqrt{M}} \varphi \mathrm{d} v \mathrm{~d} x=\left(Q_{ \pm}(h), \sqrt{M} \varphi\right)_{L_{M}^{2}}=\left(h, Q_{ \pm}(\sqrt{M} \varphi)\right)_{L_{M}^{2}}=\int_{\mathbb{R}^{4}} \frac{h}{\sqrt{M}} \frac{Q_{ \pm}(\sqrt{M} \varphi)}{\sqrt{M}} \mathrm{~d} v \mathrm{~d} x
$$

By the previous computations we know that $Q_{ \pm}(\sqrt{M} \varphi) \in L_{M}^{2} \cap \operatorname{ker} \mathcal{T}$ and thus $Q_{ \pm}(\sqrt{M} \varphi) / \sqrt{M} \in$ $L^{2} \cap \operatorname{ker} \mathcal{T}$. Since $h / \sqrt{M} \in L^{2} \cap \operatorname{ker}\langle\cdot\rangle$ we deduce that

$$
\int_{\mathbb{R}^{2}} \int_{\mathbb{R}^{2}} \frac{Q_{ \pm}(h)}{\sqrt{M}} \varphi \mathrm{d} v \mathrm{~d} x=\int_{\mathbb{R}^{2}} \int_{\mathbb{R}^{2}} \frac{h}{\sqrt{M}} \frac{Q_{ \pm}(\sqrt{M} \varphi)}{\sqrt{M}} \mathrm{~d} v \mathrm{~d} x=0
$$

saying that $\left\langle Q_{ \pm}(h) / \sqrt{M}\right\rangle=0$ and thus $Q_{ \pm}(h) \in L_{M}^{2} \cap \operatorname{ker}\langle\cdot\rangle$. 
Corollary 3.3 The average and the gain/loss collision operators are commuting on $L_{M}^{2}$.

Proof. Consider $f \in L_{M}^{2}$. Using the decomposition $f=\langle f\rangle+(f-\langle f\rangle)$ one gets by the previous lemma that $\mathcal{T} Q_{ \pm}\langle f\rangle=0,\left\langle Q_{ \pm}(f-\langle f\rangle)\right\rangle=0$ and therefore

$$
\left\langle Q_{ \pm}(f)\right\rangle=\left\langle Q_{ \pm}\langle f\rangle\right\rangle+\left\langle Q_{ \pm}(f-\langle f\rangle)\right\rangle=Q_{ \pm}\langle f\rangle .
$$

We end this section with the following commutation result, which will be used when investigating the second order approximation.

Proposition 3.15 Assume that the scattering cross section satisfies (40) and it is smooth. Then for any smooth function $f=f(x, v) \in \operatorname{ker} \mathcal{T}$ we have

$$
\begin{aligned}
\tilde{Q}^{1}(f) & :=Q\left(\tilde{\mathcal{B}} \cdot \nabla_{x, v} f\right)-\tilde{\mathcal{B}} \cdot \nabla_{x, v} Q(f) \\
& =\frac{1}{\tau} \int_{\mathbb{R}^{2}}\left(s_{1}-\frac{\left|v^{\prime}\right|}{|v|} s_{2}\right) M(v) \frac{{ }^{\perp} v}{\omega_{c}(x)} \cdot \nabla_{x} f\left(x, v^{\prime}\right) \mathrm{d} v^{\prime} \\
& +\frac{m}{\tau \theta}\left(v \cdot v_{\wedge}\right) \int_{\mathbb{R}^{2}} s_{1} M(v) f\left(x, v^{\prime}\right) \mathrm{d} v^{\prime}-\frac{m}{\tau \theta}\left(v \cdot v_{\wedge}\right) \int_{\mathbb{R}^{2}} \frac{\left|v^{\prime}\right|}{|v|} s_{2} M\left(v^{\prime}\right) f(x, v) \mathrm{d} v^{\prime}
\end{aligned}
$$

where (see Remark 3.9)

$$
\tilde{\mathcal{B}}=\left(-\frac{{ }^{\perp} v}{\omega_{c}}, v_{\wedge}-\frac{v \cdot \nabla_{x} \omega_{c}}{\omega_{c}^{2}} \perp v\right), \quad s_{1}\left(v, v^{\prime}\right)=\left\langle s\left(v, v^{\prime}\right)\right\rangle, \quad s_{2}\left(v, v^{\prime}\right)=\left\langle s\left(v, v^{\prime}\right) \frac{v \cdot v^{\prime}}{|v|\left|v^{\prime}\right|}\right\rangle .
$$

Proof. Observe that $\nabla_{v} s+\nabla_{v^{\prime}} s=0$ and therefore, performing integration by parts with respect to $v^{\prime}$ yields

$$
\begin{aligned}
\tau \tilde{\mathcal{B}} \cdot \nabla_{x, v} Q(f) & =\int_{\mathbb{R}^{2}} s\left(v, v^{\prime}\right)\left\{M(v) \tilde{\mathcal{B}}(x, v) \cdot \nabla_{x, v^{\prime}} f\left(x, v^{\prime}\right)-M\left(v^{\prime}\right) \tilde{\mathcal{B}}(x, v) \cdot \nabla_{x, v} f(x, v)\right\} \mathrm{d} v^{\prime} \\
& +\tilde{\mathcal{B}}_{v}(x, v) \cdot \int_{\mathbb{R}^{2}}\left\{\nabla_{v} M(v) f\left(x, v^{\prime}\right)-\nabla_{v^{\prime}} M\left(v^{\prime}\right) f(x, v)\right\} s\left(v, v^{\prime}\right) \mathrm{d} v^{\prime}
\end{aligned}
$$

Combining with the equality

$$
\tau Q\left(\tilde{\mathcal{B}} \cdot \nabla_{x, v} f\right)=\int_{\mathbb{R}^{2}} s\left(v, v^{\prime}\right)\left\{M(v) \tilde{\mathcal{B}}\left(x, v^{\prime}\right) \cdot \nabla_{x, v^{\prime}} f\left(x, v^{\prime}\right)-M\left(v^{\prime}\right) \tilde{\mathcal{B}}(x, v) \cdot \nabla_{x, v} f(x, v)\right\} \mathrm{d} v^{\prime}
$$

we deduce that

$$
\begin{aligned}
\tau \tilde{\mathcal{B}} \cdot \nabla_{x, v} Q(f) & -\tau Q\left(\tilde{\mathcal{B}} \cdot \nabla_{x, v} f\right)=\int_{\mathbb{R}^{2}} s M(v)\left(\tilde{\mathcal{B}}(x, v)-\tilde{\mathcal{B}}\left(x, v^{\prime}\right)\right) \cdot \nabla_{x, v^{\prime}} f\left(x, v^{\prime}\right) \mathrm{d} v^{\prime} \\
& +\tilde{\mathcal{B}}_{v}(x, v) \cdot \int_{\mathbb{R}^{2}}\left\{\nabla_{v} M(v) f\left(x, v^{\prime}\right)-\nabla_{v^{\prime}} M\left(v^{\prime}\right) f(x, v)\right\} s \mathrm{~d} v^{\prime}
\end{aligned}
$$

We transform the above terms appealing to the symmetry of $f$. Indeed, we have

$$
\begin{aligned}
T_{1} & :=\int_{\mathbb{R}^{2}} s\left(v, v^{\prime}\right) M(v)\left(\tilde{\mathcal{B}}_{x}(x, v)-\tilde{\mathcal{B}}_{x}\left(x, v^{\prime}\right)\right) \cdot \nabla_{x} f\left(x, v^{\prime}\right) \mathrm{d} v^{\prime} \\
& =\int_{\mathbb{R}^{2}} s\left(v, v^{\prime}\right) M(v) \frac{\perp\left(v^{\prime}-v\right)}{\omega_{c}(x)} \cdot \nabla_{x} f\left(x, v^{\prime}\right) \mathrm{d} v^{\prime} \\
& =\int_{\mathbb{R}^{2}} \frac{M(v)}{\omega_{c}(x)}\left\langle s\left(v, v^{\prime}\right)^{\perp}\left(v^{\prime}-v\right)\right\rangle \cdot \nabla_{x} f\left(x, v^{\prime}\right) \mathrm{d} v^{\prime} .
\end{aligned}
$$


We introduce the following averaged (with respect to $v^{\prime}$ ) scattering cross sections

$$
s_{1}\left(v, v^{\prime}\right)=\left\langle s\left(v, v^{\prime}\right)\right\rangle, s_{2}\left(v, v^{\prime}\right)=\left\langle s\left(v, v^{\prime}\right) \frac{v \cdot v^{\prime}}{|v|\left|v^{\prime}\right|}\right\rangle .
$$

Writing that $v^{\prime}=r^{\prime} \cos \alpha v /|v|-r^{\prime} \sin \alpha^{\perp} v /|v|, r^{\prime}=\left|v^{\prime}\right|, r=|v|$ one gets

$$
\begin{gathered}
v^{\prime}-v=\left(r^{\prime} \cos \alpha-r\right) \frac{v}{|v|}-r^{\prime} \sin \alpha \frac{\perp v}{|v|} \\
s_{1}\left(v, v^{\prime}\right)=\frac{1}{2 \pi} \int_{0}^{2 \pi} \sigma\left(\sqrt{\left(r^{\prime}\right)^{2}+r^{2}-2 r r^{\prime} \cos \alpha}\right) \mathrm{d} \alpha \\
s_{2}\left(v, v^{\prime}\right)=\frac{1}{2 \pi} \int_{0}^{2 \pi} \sigma\left(\sqrt{\left(r^{\prime}\right)^{2}+r^{2}-2 r r^{\prime} \cos \alpha}\right) \cos \alpha \mathrm{d} \alpha
\end{gathered}
$$

saying that $s_{1}, s_{2}$ depend only on $r=|v|, r^{\prime}=\left|v^{\prime}\right|$ and that they are symmetric with respect to $r, r^{\prime}$. Notice also that

$$
\left\langle s\left(v, v^{\prime}\right) v^{\prime}\right\rangle=\frac{1}{2 \pi} \int_{0}^{2 \pi} \sigma\left(\sqrt{\left(r^{\prime}\right)^{2}+r^{2}-2 r r^{\prime} \cos \alpha}\right)\left[r^{\prime} \cos \alpha \frac{v}{|v|}-r^{\prime} \sin \alpha \frac{\perp v}{|v|}\right] \mathrm{d} \alpha=s_{2}\left|v^{\prime}\right| \frac{v}{|v|}
$$

and

$$
\left\langle s\left(v, v^{\prime}\right)\left(v-v^{\prime}\right)\right\rangle=\left(s_{1}\left(v, v^{\prime}\right)-\frac{\left|v^{\prime}\right|}{|v|} s_{2}\left(v, v^{\prime}\right)\right) v .
$$

Therefore, the integral on the last line of (42) writes

$$
T_{1}=-\int_{\mathbb{R}^{2}}\left(s_{1}\left(v, v^{\prime}\right)-\frac{\left|v^{\prime}\right|}{|v|} s_{2}\left(v, v^{\prime}\right)\right) M(v) \frac{{ }^{\perp} v}{\omega_{c}(x)} \cdot \nabla_{x} f\left(x, v^{\prime}\right) \mathrm{d} v^{\prime} .
$$

By taking into account that ${ }^{\perp} v^{\prime} \cdot \nabla_{v^{\prime}} f\left(x, v^{\prime}\right)=0$, we observe that

$$
\begin{aligned}
T_{2} & :=\int_{\mathbb{R}^{2}} s\left(v, v^{\prime}\right) M(v)\left(\tilde{\mathcal{B}}_{v}(x, v)-\tilde{\mathcal{B}}_{v}\left(x, v^{\prime}\right)\right) \cdot \nabla_{v^{\prime}} f\left(x, v^{\prime}\right) \mathrm{d} v^{\prime} \\
& =\int_{\mathbb{R}^{2}} s\left(v, v^{\prime}\right) M(v) \frac{v \cdot \nabla_{x} \omega_{c}}{\omega_{c}^{2}} \perp\left(v^{\prime}-v\right) \cdot \nabla_{v^{\prime}} f\left(x, v^{\prime}\right) \mathrm{d} v^{\prime} \\
& =\int_{\mathbb{R}^{2}} s\left(v, v^{\prime}\right) M(v) \frac{v \cdot \nabla_{x} \omega_{c}}{\omega_{c}^{2}} \operatorname{div}_{v^{\prime}}\left\{{ }^{\perp}\left(v^{\prime}-v\right) f\left(x, v^{\prime}\right)\right\} \mathrm{d} v^{\prime} \\
& =-\int_{\mathbb{R}^{2}} \sigma^{\prime}\left(\left|v-v^{\prime}\right|\right) \frac{v^{\prime}-v}{\left|v^{\prime}-v\right|} \cdot{ }^{\perp}\left(v^{\prime}-v\right) f\left(x, v^{\prime}\right) M(v) \frac{v \cdot \nabla_{x} \omega_{c}}{\omega_{c}^{2}} \mathrm{~d} v^{\prime}=0 .
\end{aligned}
$$

For the last integral in (41) we notice that

$$
T_{3}:=\tilde{\mathcal{B}}_{v}(x, v) \cdot \int_{\mathbb{R}^{2}} \nabla_{v} M(v) f\left(x, v^{\prime}\right) s\left(v, v^{\prime}\right) \mathrm{d} v^{\prime}=-\frac{m}{\theta}\left(v \cdot v_{\wedge}\right) \int_{\mathbb{R}^{2}} s_{1}\left(v, v^{\prime}\right) M(v) f\left(x, v^{\prime}\right) \mathrm{d} v^{\prime}
$$

and

$$
\begin{aligned}
T_{4} & :=-\tilde{\mathcal{B}}_{v}(x, v) \cdot \int_{\mathbb{R}^{2}} \nabla_{v^{\prime}} M\left(v^{\prime}\right) f(x, v) s\left(v, v^{\prime}\right) \mathrm{d} v^{\prime} \\
& =\tilde{\mathcal{B}}_{v}(x, v) \cdot \int_{\mathbb{R}^{2}} \frac{m}{\theta}\left\langle s\left(v, v^{\prime}\right) v^{\prime}\right\rangle M\left(v^{\prime}\right) f(x, v) \mathrm{d} v^{\prime} \\
& =\frac{m}{\theta}\left(v_{\wedge} \cdot v\right) \int_{\mathbb{R}^{2}} s_{2}\left(v, v^{\prime}\right) \frac{\left|v^{\prime}\right|}{|v|} M\left(v^{\prime}\right) f(x, v) \mathrm{d} v^{\prime} .
\end{aligned}
$$


Finally combining (41), (43), (44), (45), (46) we obtain

$$
\begin{aligned}
\tilde{Q}^{1}(f) & =\frac{1}{\tau} \int_{\mathbb{R}^{2}}\left(s_{1}\left(v, v^{\prime}\right)-\frac{\left|v^{\prime}\right|}{|v|} s_{2}\left(v, v^{\prime}\right)\right) M(v) v \cdot\left\{\frac{m}{\theta} v_{\wedge} f\left(x, v^{\prime}\right)-\frac{\perp \nabla_{x} f\left(x, v^{\prime}\right)}{\omega_{c}(x)}\right\} \mathrm{d} v^{\prime} \\
& +\frac{1}{\tau} \int_{\mathbb{R}^{2}} s_{2}\left(v, v^{\prime}\right) \frac{\left|v^{\prime}\right|}{|v|} \frac{m}{\theta}\left(v \cdot v_{\wedge}\right)\left\{M(v) f\left(x, v^{\prime}\right)-M\left(v^{\prime}\right) f(x, v)\right\} \mathrm{d} v^{\prime} \\
& =\frac{1}{\tau} \int_{\mathbb{R}^{2}}\left(s_{1}\left(v, v^{\prime}\right)-\frac{\left|v^{\prime}\right|}{|v|} s_{2}\left(v, v^{\prime}\right)\right) M(v) F\left(x, v^{\prime}\right) \frac{{ }^{\perp} v}{\omega_{c}(x)} \cdot \nabla_{x}\left(\frac{f\left(x, v^{\prime}\right)}{F\left(x, v^{\prime}\right)}\right) \mathrm{d} v^{\prime} \\
& +\frac{1}{\tau} \int_{\mathbb{R}^{2}} s_{2}\left(v, v^{\prime}\right) \frac{\left|v^{\prime}\right|}{|v|} \frac{m}{\theta}\left(v \cdot v_{\wedge}\right) M(v) F\left(x, v^{\prime}\right)\left\{\frac{f\left(x, v^{\prime}\right)}{F\left(x, v^{\prime}\right)}-\frac{f(x, v)}{F(x, v)}\right\} \mathrm{d} v^{\prime}
\end{aligned}
$$

where $F(x, v)$ is the equilibrium of $(7)$, defined in (15).

Notice that the operator $\tilde{Q}^{1}$ has the following properties

1. the equilibrium $F$ belongs to the kernel of $\tilde{Q}^{1}$, since $\tilde{\mathcal{B}} \cdot \nabla_{x, v} F=0$ and $Q(F)=0$

$$
\tilde{Q}^{1}(F)=Q\left(\tilde{\mathcal{B}} \cdot \nabla_{x, v} F\right)-\tilde{\mathcal{B}} \cdot \nabla_{x, v} Q(F)=0 .
$$

2. $\tilde{Q}^{1}$ maps constant functions along the flow of $b^{0}$ to zero average functions since, for any smooth function $f \in \operatorname{ker} \mathcal{T}, \tilde{\mathcal{B}} \cdot \nabla_{x, v} f \in \operatorname{ker}\langle\cdot\rangle, Q(f) \in \operatorname{ker} \mathcal{T}$ cf. Lemma 3.1 and therefore

$$
Q\left(\tilde{\mathcal{B}} \cdot \nabla_{x, v} f\right) \in \operatorname{ker}\langle\cdot\rangle, \quad \tilde{\mathcal{B}} \cdot \nabla_{x, v} Q(f) \in \operatorname{ker}\langle\cdot\rangle .
$$

3. In particular $\tilde{Q}^{1}$ satisfies the local mass conservation i.e., for any smooth function $f \in \operatorname{ker} \mathcal{T}$ we have $\tilde{Q}^{1}(f) \in \operatorname{ker}\langle\cdot\rangle$ and thus

$$
\int_{\mathbb{R}^{2}} \tilde{Q}^{1}(f) \mathrm{d} v=\int_{\mathbb{R}^{2}}\left\langle\tilde{Q}^{1}(f)\right\rangle \mathrm{d} v=0
$$

4. $\tilde{Q}^{1}$ is symmetric with respect to the scalar product of $L_{F}^{2}=L^{2}\left(\frac{\mathrm{d} v \mathrm{~d} x}{F(x, v)}\right)$. Indeed, for any smooth $f, g \in \operatorname{ker} \mathcal{T}$ we have

$$
\begin{aligned}
\left(\tilde{Q}^{1}(f), g\right)_{L_{F}^{2}} & =\int_{\mathbb{R}^{2}} \int_{\mathbb{R}^{2}} Q\left(\tilde{\mathcal{B}} \cdot \nabla_{x, v} f\right) \frac{g}{F} \mathrm{~d} v \mathrm{~d} x-\int_{\mathbb{R}^{2}} \int_{\mathbb{R}^{2}} \tilde{\mathcal{B}} \cdot \nabla_{x, v} Q(f) \frac{g}{F} \mathrm{~d} v \mathrm{~d} x \\
& =\left(Q\left(\tilde{\mathcal{B}} \cdot \nabla_{x, v} f\right), g\right)_{L_{F}^{2}}+\left(Q(f), \tilde{\mathcal{B}} \cdot \nabla_{x, v} g\right)_{L_{F}^{2}} \\
& =\left(\tilde{\mathcal{B}} \cdot \nabla_{x, v} f, Q(g)\right)_{L_{F}^{2}}+\left(Q(f), \tilde{\mathcal{B}} \cdot \nabla_{x, v} g\right)_{L_{F}^{2}} \\
& =\left(\tilde{Q}^{1}(g), f\right)_{L_{F}^{2}} .
\end{aligned}
$$

5. For any smooth $f \in \operatorname{ker} \mathcal{T}$ we have $\left(\tilde{Q}^{1}(f), f\right)_{L_{F}^{2}}=0$ since $\tilde{Q}^{1}(f) \in \operatorname{ker}\langle\cdot\rangle, f / F \in \operatorname{ker} \mathcal{T}$.

Remark 3.13 When the scattering cross section is constant, we have $s_{1}=s, s_{2}=0$ and the expression of $\tilde{Q}^{1}$ becomes

$$
\tilde{Q}^{1}(f)=\frac{s}{\tau} M(v) \rho_{F}(x) \frac{{ }^{\perp} v}{\omega_{c}(x)} \cdot \nabla_{x}\left(\frac{\rho_{f}}{\rho_{F}}\right)
$$

where $\rho_{f}(x)=\int_{\mathbb{R}^{2}} f\left(x, v^{\prime}\right) \mathrm{d} v^{\prime}, \rho_{F}(x)=\int_{\mathbb{R}^{2}} F\left(x, v^{\prime}\right) \mathrm{d} v^{\prime}=\exp (-q \phi(x) / \theta)$. 


\section{Asymptotic models for Boltzmann equation}

This section is devoted to the derivation of the limit model for (7) when $\varepsilon$ becomes very small. At the formal level, we perform our analysis starting from the expansion $f^{\varepsilon}=$ $f+\varepsilon f^{1}+\varepsilon^{2} f^{2}+\ldots$. We are interested in first order but also second order asymptotic models. We present the weak and strong convergence results that have been announced in Theorems $2.1,2.2,2.3$.

\subsection{First order model}

We investigate the asymptotic behaviour of the linear Boltzmann problem (7), (2) when $\varepsilon \searrow 0$. At least formally, the leading order term $f$ satisfies (9) and the constraint $\mathcal{T} f(t)=$ $0, t \in \mathbb{R}_{+}$. By Proposition 3.2 we know that Range $\mathcal{T}=\operatorname{ker}\langle\cdot\rangle$ and therefore (9) is equivalent to

$$
\left\langle\partial_{t} f+v \cdot \nabla_{x} f+\frac{q}{m} E \cdot \nabla_{v} f\right\rangle=\langle Q(f)\rangle .
$$

It is easily seen that $\left\langle\partial_{t} f\right\rangle=\partial_{t}\langle f\rangle=\partial_{t} f$ and by Corollary 3.3 we know that $\langle Q(f)\rangle=$ $Q(\langle f\rangle)=Q(f)$. Moreover, there is a function $g$ such that $f(t, x, v)=g(t, x, r=|v|)$ and therefore

$$
\left\langle v \cdot \nabla_{x} f\right\rangle=\langle v\rangle \cdot \nabla_{x} g=0,\left\langle E \cdot \nabla_{v} f\right\rangle=\left\langle E \cdot \frac{v}{|v|} \partial_{r} g\right\rangle=\frac{\langle v\rangle}{|v|} \cdot E \partial_{r} g=0
$$

since $\langle v\rangle=0$. We obtain the limit model

$$
\partial_{t} f=Q(f), \quad \mathcal{T} f=0
$$

which can be justified rigorously at least under the confinement potential hypothesis (14). Indeed, in this case $F(x, v)$ belongs to $L_{F}^{2}$ and solves (7) for any $\varepsilon>0$. Notice that $Q_{ \pm}$are also bounded from $L_{F}^{2}$ to $L_{F}^{2}$. It is well known that for any convex function $H$, the relative entropy

$$
\mathcal{H}^{\varepsilon}(t)=\int_{\mathbb{R}^{2}} \int_{\mathbb{R}^{2}} F(x, v) H\left(\frac{f^{\varepsilon}(t, x, v)}{F(x, v)}\right) \mathrm{d} v \mathrm{~d} x
$$

satisfies

$$
\frac{d \mathcal{H}^{\varepsilon}}{d t}+\mathcal{D}^{\varepsilon}(t)=0, \quad t \in \mathbb{R}_{+}
$$

where the dissipative term $\mathcal{D}^{\varepsilon}$ is given by

$$
\mathcal{D}^{\varepsilon}(t)=\frac{1}{\tau} \int_{\mathbb{R}^{2}} \int_{\mathbb{R}^{2}} \int_{\mathbb{R}^{2}} s\left(v, v^{\prime}\right) M(v) F\left(x, v^{\prime}\right) D\left(\frac{f^{\varepsilon}(t, x, v)}{F(x, v)}, \frac{f^{\varepsilon}\left(t, x, v^{\prime}\right)}{F\left(x, v^{\prime}\right)}\right) \mathrm{d} v^{\prime} \mathrm{d} v \mathrm{~d} x
$$

where

$$
D\left(u, u^{\prime}\right)=H\left(u^{\prime}\right)-H(u)-\left(u^{\prime}-u\right) H^{\prime}(u) \geq 0, \quad u, u^{\prime} \in \mathbb{R} .
$$

Assuming that the initial condition satisfies

$$
f^{\text {in }} \geq 0, f^{\text {in }} \in L_{F}^{2}
$$

one gets by (47) the stability inequality (16) which allows us to pass to the limit, at least weakly, in (7). 
Proof. (of Theorem 2.1) We have the uniform bound

$$
\sup _{\varepsilon>0}\left\|f^{\varepsilon}\right\|_{L^{\infty}\left(\mathbb{R}_{+} ; L_{F}^{2}\right)} \leq\left\|f^{\text {in }}\right\|_{L_{F}^{2}}
$$

and we can assume (after extraction of a sequence $\varepsilon_{k} \searrow 0$ )

$$
\lim _{k \rightarrow+\infty} f^{\varepsilon_{k}}=f, \quad \text { weakly } \star \text { in } L^{\infty}\left(\mathbb{R}_{+} ; L_{F}^{2}\right) .
$$

Using the weak formulation (7) it is easily seen that the weak limit $f$ satisfies the constraint $\mathcal{T} f=0$. Taking now test functions of the form $\eta(t) \varphi(x, v), \eta \in C_{c}^{1}\left(\mathbb{R}_{+}\right), \varphi \in C_{c}^{1}\left(\mathbb{R}^{2} \times \mathbb{R}^{2}\right) \cap$ $\operatorname{ker} \mathcal{T}$ (the constraint $\varphi \in \operatorname{ker} \mathcal{T}$ is imposed in order to remove the singular term $\frac{1}{\varepsilon} \mathcal{T} f^{\varepsilon}$ in (7)) we obtain

$$
\begin{aligned}
-\int_{\mathbb{R}_{+}} \int_{\mathbb{R}^{2}} \int_{\mathbb{R}^{2}} f^{\varepsilon_{k}}\left(\eta^{\prime} \varphi+\eta v \cdot \nabla_{x} \varphi+\eta \frac{q}{m} E \cdot \nabla_{v} \varphi\right) \mathrm{d} v \mathrm{~d} x \mathrm{~d} t & -\int_{\mathbb{R}^{2}} \int_{\mathbb{R}^{2}} f^{\mathrm{in}} \eta(0) \varphi \mathrm{d} v \mathrm{~d} x \\
& =\int_{\mathbb{R}_{+}} \int_{\mathbb{R}^{2}} \int_{\mathbb{R}^{2}} Q\left(f^{\varepsilon_{k}}\right) \eta \varphi \mathrm{d} v \mathrm{~d} x \mathrm{~d} t .
\end{aligned}
$$

By the symmetry of the collision operator one gets, noticing that $\eta Q(F \varphi) \in L^{1}\left(\mathbb{R}_{+} ; L_{F}^{2}\right)$

$$
\begin{aligned}
\lim _{k \rightarrow+\infty} \int_{\mathbb{R}_{+}} \int_{\mathbb{R}^{2}} \int_{\mathbb{R}^{2}} Q\left(f^{\varepsilon_{k}}\right) \eta \varphi \mathrm{d} v \mathrm{~d} x \mathrm{~d} t & =\lim _{k \rightarrow+\infty} \int_{\mathbb{R}_{+}}\left(f^{\varepsilon_{k}}(t), \eta(t) Q(F \varphi)\right)_{L_{F}^{2}} \mathrm{~d} t \\
& =\int_{\mathbb{R}_{+}}(f(t), \eta(t) Q(F \varphi))_{L_{F}^{2}} \mathrm{~d} t \\
& =\int_{\mathbb{R}_{+}} \int_{\mathbb{R}^{2}} \int_{\mathbb{R}^{2}} Q(f) \eta \varphi \mathrm{d} v \mathrm{~d} x \mathrm{~d} t .
\end{aligned}
$$

Passing to the limit with respect to $k \rightarrow+\infty$ in (50) leads to the weak formulation

$$
\begin{aligned}
-\int_{\mathbb{R}_{+}} \int_{\mathbb{R}^{2}} \int_{\mathbb{R}^{2}} f\left(\eta^{\prime} \varphi+\eta v \cdot \nabla_{x} \varphi+\eta \frac{q}{m} E \cdot \nabla_{v} \varphi\right) \mathrm{d} v \mathrm{~d} x \mathrm{~d} t & -\int_{\mathbb{R}^{2}} \int_{\mathbb{R}^{2}} f^{\mathrm{in}} \eta(0) \varphi \mathrm{d} v \mathrm{~d} x \\
& =\int_{\mathbb{R}_{+}} \int_{\mathbb{R}^{2}} \int_{\mathbb{R}^{2}} Q(f) \eta \varphi \mathrm{d} v \mathrm{~d} x \mathrm{~d} t
\end{aligned}
$$

for $\eta \in C_{c}^{1}\left(\mathbb{R}_{+}\right), \varphi \in C_{c}^{1}\left(\mathbb{R}^{2} \times \mathbb{R}^{2}\right) \cap \operatorname{ker} \mathcal{T}$. Writing $\varphi(x, v)=\psi(x,|v|)$ we deduce that $\left\langle v \cdot \nabla_{x} \varphi\right\rangle=0,\left\langle E \cdot \nabla_{v} \varphi\right\rangle=0$ and therefore, since $\mathcal{T} f=0$, one gets

$$
\int_{\mathbb{R}_{+}} \int_{\mathbb{R}^{2}} \int_{\mathbb{R}^{2}} f\left(\eta v \cdot \nabla_{x} \varphi+\eta \frac{q}{m} E \cdot \nabla_{v} \varphi\right) \mathrm{d} v \mathrm{~d} x \mathrm{~d} t=0 .
$$

Finally the formulation (51) reduces to

$$
-\int_{\mathbb{R}_{+}} \int_{\mathbb{R}^{2}} \int_{\mathbb{R}^{2}} f \partial_{t}(\eta \varphi) \mathrm{d} v \mathrm{~d} x \mathrm{~d} t-\int_{\mathbb{R}^{2}} \int_{\mathbb{R}^{2}}\left\langle f^{\mathrm{in}}\right\rangle \eta(0) \varphi \mathrm{d} v \mathrm{~d} x=\int_{\mathbb{R}_{+}} \int_{\mathbb{R}^{2}} \int_{\mathbb{R}^{2}} Q(f) \eta \varphi \mathrm{d} v \mathrm{~d} x \mathrm{~d} t
$$

for test functions satisfying the constraint $\mathcal{T}(\eta \varphi)=0$ at any time $t \in \mathbb{R}_{+}$. Since $f,\left\langle f^{\text {in }}\right\rangle$ and $Q(f)$ also satisfy this constraint it is easily seen that $f$ solves weakly (17). By the uniqueness of the solution for (17) we deduce that all the family $\left(f^{\varepsilon}\right)_{\varepsilon>0}$ converges weakly $\star$ in $L^{\infty}\left(\mathbb{R}_{+} ; L_{F}^{2}\right)$ towards $f$. It remains to justify that the constraint $\mathcal{T} f(t)=0$ is automatically satisfied at any time $t>0$. Indeed, for any $\alpha>0$ we have, cf. Lemma 3.1

$$
\partial_{t} f_{\alpha}=\left(\partial_{t} f\right)\left(x, R_{\alpha} v\right)=(Q f)\left(x, R_{\alpha} v\right)=\left(Q f_{\alpha}\right)(x, v) .
$$

Since $f_{\alpha}(0, x, v)=\left\langle f^{\text {in }}\right\rangle\left(x, R_{\alpha} v\right)=\left\langle f^{\text {in }}\right\rangle(x, v)=f(0, x, v)$ we deduce that both $f$ and $f_{\alpha}$ solve (17) and, by the uniqueness of the solution, one gets $f_{\alpha}(t)=f(t)$ for any $\alpha \in \mathbb{R}$, saying that $\mathcal{T} f(t)=0$ at any time $t>0$. 
Under appropriate smoothness hypotheses on the initial condition $f^{\text {in }}$ and the electromagnetic field, it is possible to obtain strong convergence results. Actually we can show that $f^{\varepsilon}=f+\mathcal{O}(\varepsilon)$ strongly, which justifies the first term in the expansion $f^{\varepsilon}=f+\varepsilon f^{1}+\ldots$. We only sketch the main lines of the arguments and skip the standard details.

Proof. (of Theorem 2.2) The main idea is to introduce a first order correction term. Assume for the moment that the dominant term $f$ is smooth enough (with respect to all variables). Since

$$
\left\langle\partial_{t} f+v \cdot \nabla_{x} f+\frac{q}{m} E \cdot \nabla_{v} f-Q(f)\right\rangle=0
$$

we deduce by Proposition 3.2 that there is a unique function $f^{1}$ such that at any time $t>0$

$$
\partial_{t} f+v \cdot \nabla_{x} f+\frac{q}{m} E \cdot \nabla_{v} f+\mathcal{T} f^{1}=Q(f),\left\langle f^{1}\right\rangle=0 .
$$

Combining with (7) we obtain

$$
\left(\partial_{t}+v \cdot \nabla_{x}+\frac{q}{m} E \cdot \nabla_{v}+\frac{\mathcal{T}}{\varepsilon}\right)\left(f^{\varepsilon}-f-\varepsilon f^{1}\right)=Q\left(f^{\varepsilon}-f-\varepsilon f^{1}\right)+\varepsilon \mathcal{R}\left(f^{1}\right)
$$

where

$$
\mathcal{R}\left(f^{1}\right)=-\left(\partial_{t}+v \cdot \nabla_{x}+\frac{q}{m} E \cdot \nabla_{v}\right) f^{1}+Q\left(f^{1}\right) .
$$

Notice that $\mathcal{R}\left(f^{1}\right)$ is of order of 1 , provided that $f^{1}$ is smooth enough (we will see later on how we can get regularity for $f^{1}$ from the regularity of $f$ ). Assume for the moment that $f^{1}$ is smooth enough. In this case $\varepsilon \mathcal{R}\left(f^{1}\right)$ will be of order of $\varepsilon$. Using again the relative entropy dissipation (similar to (47), since now we have an extra term $\varepsilon \mathcal{R}\left(f^{1}\right)$ ) or multiplying (53) by $\left(f^{\varepsilon}-f-\varepsilon f^{1}\right) / F$, where $F$ is the equilibrium of $(7)$, one gets

$$
\begin{aligned}
\frac{1}{2} \frac{\mathrm{d}}{\mathrm{d} t}\left\|\left(f^{\varepsilon}-f-\varepsilon f^{1}\right)(t)\right\|_{L_{F}^{2}}^{2} & \leq \varepsilon \int_{\mathbb{R}^{2}} \int_{\mathbb{R}^{2}} \mathcal{R}\left(f^{1}\right) \frac{f^{\varepsilon}-f-\varepsilon f^{1}}{F} \mathrm{~d} v \mathrm{~d} x \\
& \leq \varepsilon\left\|\mathcal{R}\left(f^{1}(t)\right)\right\|_{L_{F}^{2}}\left\|\left(f^{\varepsilon}-f-\varepsilon f^{1}\right)(t)\right\|_{L_{F}^{2}}
\end{aligned}
$$

implying that

$$
\frac{\mathrm{d}}{\mathrm{d} t}\left\|\left(f^{\varepsilon}-f-\varepsilon f^{1}\right)(t)\right\|_{L_{F}^{2}} \leq \varepsilon\left\|\mathcal{R}\left(f^{1}(t)\right)\right\|_{L_{F}^{2}}
$$

Finally one gets

$$
\left\|\left(f^{\varepsilon}-f-\varepsilon f^{1}\right)(t)\right\|_{L_{F}^{2}} \leq\left\|f^{\mathrm{in}}-\left\langle f^{\mathrm{in}}\right\rangle-\varepsilon f^{1}(0)\right\|_{L_{F}^{2}}+\varepsilon \int_{0}^{t}\left\|\mathcal{R}\left(f^{1}(s)\right)\right\|_{L_{F}^{2}} \mathrm{~d} s
$$

and therefore, for any $T>0$, there is a constant $C_{T}$ depending on $\left\|f^{1}\right\|_{L^{\infty}\left(\mathbb{R}_{+} ; L_{F}^{2}\right)}$ and $\left\|\mathcal{R}\left(f^{1}\right)\right\|_{L^{1}\left(0, T ; L_{F}^{2}\right)}$ such that

$$
\left\|\left(f^{\varepsilon}-f\right)(t)\right\|_{L_{F}^{2}} \leq C_{T} \varepsilon, \quad t \in[0, T], \quad \varepsilon>0
$$

provided that the initial condition is well prepared i.e., $\mathcal{T} f^{\text {in }}=0$.

It remains to justify the smoothness of $f$ and $f^{1}$.

Proposition 4.1 Assume that the initial condition satisfies $f^{\text {in }},|v| f^{\text {in }} \in L_{F}^{2}, \mathcal{T} f^{\text {in }}=0$. Then the solution of (17) satisfies $f \in L^{\infty}\left(\mathbb{R}_{+} ; L_{F}^{2}\right)$, vf $\in L^{\infty}\left(\mathbb{R}_{+} ; L_{F}^{2}\right)$. 
Proof. We know that $\|f(t)\|_{L_{F}^{2}} \leq\left\|f^{\text {in }}\right\|_{L_{F}^{2}}$. Multiplying the equation in (17) by $v$ one gets

$$
\partial_{t}(v f)+v f(t, x, v) \frac{1}{\tau} \int_{\mathbb{R}^{2}} s\left(v, v^{\prime}\right) M\left(v^{\prime}\right) \mathrm{d} v^{\prime}=v M(v) \frac{1}{\tau} \int_{\mathbb{R}^{2}} s\left(v, v^{\prime}\right) f\left(t, x, v^{\prime}\right) \mathrm{d} v^{\prime} .
$$

Solving the above differential equation with respect to $v f$ and estimating the right hand side by using

$$
\int_{\mathbb{R}^{2}} s\left(v, v^{\prime}\right) f\left(t, x, v^{\prime}\right) \mathrm{d} v^{\prime} \leq S_{0}\left\|f^{\mathrm{in}}\right\|_{L_{M}^{2}}
$$

lead to the bound

$$
\|v f(t)\|_{L_{F}^{2}} \leq\left\|v f^{\mathrm{in}}\right\|_{L_{F}^{2}}+\frac{S_{0}}{s_{0}}\left(\int_{\mathbb{R}^{2}}\left|v^{\prime}\right|^{2} M\left(v^{\prime}\right) \mathrm{d} v^{\prime}\right)^{1 / 2}\left\|f^{\mathrm{in}}\right\|_{L_{F}^{2}}, \quad t>0 .
$$

Proposition 4.2 Assume that the function $\sigma$, introduced in (40), is Lipschitz continuous and that the initial condition satisfies

$$
\mathcal{T} f^{\text {in }}=0, \quad(1+|v|)\left(\left|f^{\text {in }}\right|+\left|\nabla_{x} f^{\text {in }}\right|+\left|\nabla_{v} f^{\text {in }}\right|\right) \in L_{F}^{2} .
$$

Then the solution of (17) satisfies

$$
(1+|v|)\left(|f|+\left|\partial_{t} f\right|+\left|\nabla_{x} f\right|+\left|\nabla_{v} f\right|\right) \in L^{\infty}\left(\mathbb{R}_{+} ; L_{F}^{2}\right) .
$$

Moreover, if $\sigma \in W^{2, \infty}$ and $\left(1+|v|^{2}\right) \nabla_{x, v}^{2} f^{\text {in }} \in L_{F}^{2}$ then $\left(1+|v|^{2}\right) \nabla_{t, x, v}^{2} f \in L^{\infty}\left(\mathbb{R}_{+} ; L_{F}^{2}\right)$.

Proof. By Proposition 4.1 we know that $(1+|v|) f \in L^{\infty}\left(\mathbb{R}_{+} ; L_{F}^{2}\right)$ and since the operators $Q_{ \pm}$remain bounded in $L_{F}^{2}$ (see Proposition 3.14 )

$$
\|Q(f)\|_{L_{F}^{2}} \leq \frac{2 S_{0}}{\tau}\|f\|_{L_{F}^{2}}
$$

one gets $\left\|\partial_{t} f\right\|_{L^{\infty}\left(\mathbb{R}_{+} ; L_{F}^{2}\right)} \leq \frac{2 S_{0}}{\tau}\left\|f^{\text {in }}\right\|_{L_{F}^{2}}$. Observe that

$$
\int_{\mathbb{R}^{2}} s\left(v, v^{\prime}\right) f\left(t, x, v^{\prime}\right) \mathrm{d} v^{\prime} \leq\left(\int_{\mathbb{R}^{2}} s\left(v, v^{\prime}\right)^{2} M\left(v^{\prime}\right) \mathrm{d} v^{\prime}\right)^{1 / 2}\|f(t, x, \cdot)\|_{L_{M}^{2}} \leq S_{0}\left\|f^{\mathrm{in}}(x, \cdot)\right\|_{L_{M}^{2}} .
$$

Therefore we obtain

$$
\left\|v \partial_{t} f\right\|_{L_{F}^{2}}=\|v Q(f)\|_{L_{F}^{2}} \leq \frac{S_{0}}{\tau}\left(\int_{\mathbb{R}^{2}}\left|v^{\prime}\right|^{2} M\left(v^{\prime}\right) \mathrm{d} v^{\prime}\right)^{1 / 2}\left\|f^{\mathrm{in}}\right\|_{L_{F}^{2}}+\frac{S_{0}}{\tau}\|v f(t)\|_{L_{F}^{2}}
$$

saying that $v \partial_{t} f \in L^{\infty}\left(\mathbb{R}_{+} ; L_{F}^{2}\right)$. Since $Q$ is commuting with the space derivatives one gets

$$
\partial_{t}\left(\nabla_{x} f\right)=Q\left(\nabla_{x} f\right), \quad(t, x, v) \in \mathbb{R}_{+}^{\star} \times \mathbb{R}^{2} \times \mathbb{R}^{2}, \quad \nabla_{x} f(0)=\nabla_{x} f^{\text {in }} \in \operatorname{ker} \mathcal{T}
$$

and therefore, by Proposition 4.1 we obtain $(1+|v|)\left|\nabla_{x} f\right| \in L^{\infty}\left(\mathbb{R}_{+} ; L_{F}^{2}\right)$. It remains to estimate the velocity derivatives. As we already know that ${ }^{\perp} v \cdot \nabla_{v} f=0$ let us analyze the derivative along $v$. We have

$$
\partial_{t}\left(\frac{v}{|v|} \cdot \nabla_{v} f\right)+\frac{1}{\tau}\left(\frac{v}{|v|} \cdot \nabla_{v} f\right) \int_{\mathbb{R}^{2}} s\left(v, v^{\prime}\right) M\left(v^{\prime}\right) \mathrm{d} v^{\prime}=\frac{1}{\tau} g
$$


where

$g=\int_{\mathbb{R}^{2}} \frac{v}{|v|} \cdot \nabla_{v} s\left\{M(v) f\left(t, x, v^{\prime}\right)-M\left(v^{\prime}\right) f(t, x, v)\right\} \mathrm{d} v^{\prime}+\frac{v}{|v|} \cdot \nabla_{v} M(v) \int_{\mathbb{R}^{2}} s\left(v, v^{\prime}\right) f\left(t, x, v^{\prime}\right) \mathrm{d} v^{\prime}$.

Observe that

$$
\|g\|_{L_{F}^{2}} \leq 2\left\|\sigma^{\prime}\right\|_{L^{\infty}}\left\|f^{\mathrm{in}}\right\|_{L_{F}^{2}}+S_{0}\left\|f^{\mathrm{in}}\right\|_{L_{F}^{2}}\left\|\frac{v}{|v|} \cdot \nabla_{v} M\right\|_{L_{M}^{2}}
$$

and finally, solving (55) with respect to $\frac{v}{|v|} \cdot \nabla_{v} f$ yields

$$
\left\|\frac{v}{|v|} \cdot \nabla_{v} f(t)\right\|_{L_{F}^{2}} \leq\left\|\frac{v}{|v|} \cdot \nabla_{v} f^{\mathrm{in}}\right\|_{L_{F}^{2}}+\frac{1}{s_{0}}\left(2\left\|\sigma^{\prime}\right\|_{L^{\infty}}+S_{0}\left\|\frac{v}{|v|} \cdot \nabla_{v} M\right\|_{L_{M}^{2}}\right)\left\|f^{\mathrm{in}}\right\|_{L_{F}^{2}} .
$$

Therefore $\nabla_{v} f \in L^{\infty}\left(\mathbb{R}_{+} ; L_{F}^{2}\right)$. Starting from the equality $\partial_{t}\left(v \cdot \nabla_{v} f\right)=v \cdot \nabla_{v} Q(f)$ we obtain the differential equation

$$
\partial_{t}\left(v \cdot \nabla_{v} f\right)+\frac{1}{\tau}\left(v \cdot \nabla_{v} f\right) \int_{\mathbb{R}^{2}} s\left(v, v^{\prime}\right) M\left(v^{\prime}\right) \mathrm{d} v^{\prime}=\frac{1}{\tau} h
$$

where

$$
h=\int_{\mathbb{R}^{2}} v \cdot \nabla_{v} s\left\{M(v) f\left(t, x, v^{\prime}\right)-M\left(v^{\prime}\right) f(t, x, v)\right\} \mathrm{d} v^{\prime}+v \cdot \nabla_{v} M \int_{\mathbb{R}^{2}} s\left(v, v^{\prime}\right) f\left(t, x, v^{\prime}\right) \mathrm{d} v^{\prime} .
$$

Observing that

$$
\|h(t)\|_{L_{F}^{2}} \leq\left\|\sigma^{\prime}\right\|_{L^{\infty}}\|v M\|_{L_{M}^{2}}\left\|f^{\mathrm{in}}\right\|_{L_{F}^{2}}+\left\|\sigma^{\prime}\right\|_{L^{\infty}}\|v f(t)\|_{L_{F}^{2}}+S_{0}\left\|v \cdot \nabla_{v} M\right\|_{L_{M}^{2}}\left\|f^{\mathrm{in}}\right\|_{L_{F}^{2}}
$$

we deduce that $\left\|v \cdot \nabla_{v} f(t)\right\|_{L_{F}^{2}} \leq\left\|v \cdot \nabla_{v} f^{\text {in }}\right\|_{L_{F}^{2}}+1 / s_{0}\|h\|_{L^{\infty}\left(\mathbb{R}_{+} ; L_{F}^{2}\right)}$ which says that $|v|\left|\nabla_{v} f\right|=$ $\left|v \cdot \nabla_{v} f\right| \in L^{\infty}\left(\mathbb{R}_{+} ; L_{F}^{2}\right)$. The last statement follows similarly.

Proposition 4.3 Assume that the hypotheses of Propositions 3.2 and 4.2 hold true and that the electro-magnetic field $(E(x), B(x))$ belongs to $W^{1, \infty}\left(\mathbb{R}^{2}\right)$. Then we have

$$
f^{1} \in L^{\infty}\left(\mathbb{R}_{+} ; L_{F}^{2}\right), \quad \mathcal{R}\left(f^{1}\right) \in L^{\infty}\left(\mathbb{R}_{+} ; L_{F}^{2}\right)
$$

where $f^{1}$ is defined by (52) and $\mathcal{R}\left(f^{1}\right)$ by $(54)$.

Proof. Since $\partial_{t} f=Q(f)$, notice that (52) can be written

$$
v \cdot \nabla_{x} f+\frac{q}{m} E(x) \cdot \nabla_{v} f+\mathcal{T} f^{1}=0,\left\langle f^{1}\right\rangle=0
$$

and therefore we have, cf. Remark 3.9

$$
f^{1}=-\mathcal{B} \cdot \nabla_{x, v} f=\frac{{ }^{\perp} v}{\omega_{c}(x)} \cdot \nabla_{x} f-\frac{q}{m \omega_{c}(x)}{ }^{\perp} E \cdot \nabla_{v} f .
$$

Our conclusions follow easily by the smoothness of $f$.

Combining the previous arguments we have proved the strong convergence result stated in Theorem 2.2. 


\subsection{Second order model}

As we have seen in the previous section, the leading order term in the expansion $f^{\varepsilon}=$ $f+\varepsilon f^{1}+\ldots$ satisfies the space homogeneous Boltzmann equation. The collision operator appearing in the first order limit model is still the linear Boltzmann operator. In other words the magnetic field does not affect the collision mechanism (the collision operator and the advection along the Laplace force $\omega_{c}^{\perp} v \cdot \nabla_{v}$ are commuting). Our goal in this section is to take into account the first order correction. We are looking for a closure involving $f+\varepsilon f^{1}$ accounting for the small departures from the equilibrium $f$ when the magnetic field grows like $\mathcal{O}(1 / \varepsilon)$. We expect that the magnetic field will affect the collision mechanism. Naturally, the new collision operator should verify the usual physical properties like mass conservation, entropy dissipation. The limit model being quite complicated we prefer to skip the technical details leading eventually to rigorous results. Nevertheless we expect that the arguments employed when studying the first order model will adapt to the second order one as well.

The linear Boltzmann equation (7) reads

$$
\partial_{t} f^{\varepsilon}+a \cdot \nabla_{x, v} f^{\varepsilon}+\frac{1}{\varepsilon} \mathcal{T} f^{\varepsilon}=Q\left(f^{\varepsilon}\right)
$$

where $a=(v, q / m E(x)), \mathcal{T}=\omega_{c}(x)^{\perp} v \cdot \nabla_{v}, \omega_{c}(x)=q B(x) / m$. Plugging the expansion $f^{\varepsilon}=f+\varepsilon f^{1}+\ldots$ in (56) yields the constraint $\mathcal{T} f=0$ and the evolution equations

$$
\begin{gathered}
\partial_{t} f+a \cdot \nabla_{x, v} f+\mathcal{T} f^{1}=Q(f) \\
\partial_{t} f^{1}+a \cdot \nabla_{x, v} f^{1}+\mathcal{T} f^{2}=Q\left(f^{1}\right)
\end{gathered}
$$

The evolution equation for the dominant term $f$ has been obtained by averaging (57) along the flow of $\mathcal{T}$. Indeed, by Proposition 3.10 we know that there is a vector field $\mathcal{A}=\mathcal{A}(x, v)$ such that

$$
\left\langle a \cdot \nabla_{x, v} f\right\rangle=\mathcal{A} \cdot \nabla_{x, v} f, \quad f \in \operatorname{ker} \mathcal{T} .
$$

Actually, in the two dimensional setting, the average field associated to $a=(v, q / m E(x))$ is $\mathcal{A}=0$ cf. Remark 3.6, but we prefer to keep track of the vector field $\mathcal{A}$, which will clearly show that our technique will provide a second order closure regardless of whether the field $\mathcal{A}$ vanishes or not (this emphasizes how to obtain the second order closure in the three dimensional setting, in which case $\mathcal{A} \neq 0$ ). Nevertheless, in the final two dimensional model the field $\mathcal{A}$ will be replaced by its value $\mathcal{A}=0$. For any $f \in \operatorname{ker} \mathcal{T}$ we have $\left\langle\partial_{t} f\right\rangle=\partial_{t}\langle f\rangle=\partial_{t} f$ and by Corollary 3.3 we can write

$$
\langle Q(f)\rangle=Q(\langle f\rangle)=Q(f) .
$$

Therefore averaging (57) leads to the Boltzmann equation

$$
\partial_{t} f+\mathcal{A} \cdot \nabla_{x, v} f=Q(f) .
$$

Clearly more information is available from (57). In order to exploit that we use the decomposition

$$
f^{1}=g^{1}+h^{1}, \quad g^{1}=\left\langle f^{1}\right\rangle \in \operatorname{ker} \mathcal{T}, \quad h^{1}=f^{1}-\left\langle f^{1}\right\rangle \in \operatorname{ker}\langle\cdot\rangle
$$

and therefore (57) becomes

$$
\partial_{t} f+a \cdot \nabla_{x, v} f+\mathcal{T} h^{1}=Q(f)
$$


The equations (59), (60) yields

$$
(a-\mathcal{A}) \cdot \nabla_{x, v} f+\mathcal{T} h^{1}=0
$$

and by Remark 3.9 we deduce that $h^{1}=-\tilde{\mathcal{B}} \cdot \nabla_{x, v} f$ where the field $\tilde{\mathcal{B}}$ writes (see Remark $3.9)$

$$
\tilde{\mathcal{B}}=\frac{1}{\omega_{c}(x)}\left(-{ }^{\perp} v, \frac{q}{m}{ }^{\perp} E-\frac{v \cdot \nabla_{x} \omega_{c}}{\omega_{c}(x)}{ }^{\perp} v\right) .
$$

We average now (58). Notice that we have

$$
\begin{gathered}
\left\langle\partial_{t} f^{1}\right\rangle=\partial_{t}\left\langle f^{1}\right\rangle=\partial_{t} g^{1},\left\langle\mathcal{T} f^{2}\right\rangle=0,\left\langle Q\left(f^{1}\right)\right\rangle=Q\left(\left\langle f^{1}\right\rangle\right)=Q\left(g^{1}\right) \\
\left\langle a \cdot \nabla_{x, v} f^{1}\right\rangle=\left\langle a \cdot \nabla_{x, v} g^{1}\right\rangle+\left\langle a \cdot \nabla_{x, v} h^{1}\right\rangle=\mathcal{A} \cdot \nabla_{x, v} g^{1}-\left\langle a \cdot \nabla_{x, v}\left(\tilde{\mathcal{B}} \cdot \nabla_{x, v} f\right)\right\rangle
\end{gathered}
$$

and therefore we obtain the equation

$$
\partial_{t} g^{1}+\mathcal{A} \cdot \nabla_{x, v} g^{1}-\left\langle a \cdot \nabla_{x, v}\left(\tilde{\mathcal{B}} \cdot \nabla_{x, v} f\right)\right\rangle=Q\left(g^{1}\right) .
$$

Naturally, the closure for $f+\varepsilon f^{1}=f+\varepsilon g^{1}+\varepsilon h^{1}$ comes by combining (59), (62)

$$
\left(\partial_{t}+\mathcal{A} \cdot \nabla_{x, v}\right)\left(f+\varepsilon f^{1}\right)+\varepsilon\left(\left\langle a \cdot \nabla_{x, v}\right\rangle-\mathcal{A} \cdot \nabla_{x, v}+Q-\partial_{t}\right) h^{1}=Q\left(f+\varepsilon f^{1}\right) .
$$

Making use of Remark 3.12 and Proposition 3.15 we obtain

$$
\begin{aligned}
\left(\left\langle a \cdot \nabla_{x, v}\right\rangle-\mathcal{A} \cdot \nabla_{x, v}\right. & \left.+Q-\partial_{t}\right) h^{1}=-\operatorname{div}_{x, v}(f \mathcal{C})-Q\left(\tilde{\mathcal{B}} \cdot \nabla_{x, v} f\right)+\partial_{t}\left(\tilde{\mathcal{B}} \cdot \nabla_{x, v} f\right) \\
& =-\operatorname{div}_{x, v}(f \mathcal{C})+\tilde{\mathcal{B}} \cdot \nabla_{x, v} Q(f)-Q\left(\tilde{\mathcal{B}} \cdot \nabla_{x, v} f\right) \\
& =-\mathcal{C} \cdot \nabla_{x, v} f-\tilde{Q}^{1}(f) .
\end{aligned}
$$

Finally (63) becomes

$$
\left(\partial_{t}+(\mathcal{A}-\varepsilon \mathcal{C}) \cdot \nabla_{x, v}\right)\left(f+\varepsilon f^{1}\right)=Q\left(f+\varepsilon f^{1}\right)+\varepsilon \tilde{Q}^{1}(f)-\varepsilon^{2} \mathcal{C} \cdot \nabla_{x, v} f^{1}
$$

and since we are looking for a closure with respect to $f+\varepsilon f^{1}$, or something equivalent to $f+\varepsilon f^{1}$ up to a second order term $\mathcal{O}\left(\varepsilon^{2}\right)$, we need to define $\tilde{Q}^{1}$ over the space of all functions (not only on the subspace of constant functions along the flow of $b^{0}$, as it was done in Proposition 3.15). We denote by $Q^{1}$ this extension and assume for the moment that $Q^{1}$ is known (the exact expression will be determined in Proposition 4.4 and Corollary 4.1). By doing that we observe that the right hand side of (64) writes

$$
\left(Q+\varepsilon Q^{1}\right)\left(f+\varepsilon f^{1}\right)-\varepsilon^{2}\left(\mathcal{C} \cdot \nabla_{x, v} f^{1}+Q^{1}\left(f^{1}\right)\right) .
$$

Getting rid of the second order term and taking into account that $\mathcal{A}=0$ (in the two dimensional setting), we may expect that solving

$$
\partial_{t} \tilde{f}^{\varepsilon}-\varepsilon \mathcal{C} \cdot \nabla_{x, v} \tilde{f}^{\varepsilon}=\left(Q+\varepsilon Q^{1}\right)\left(\tilde{f}^{\varepsilon}\right)
$$

provides a second order approximation of $f^{\varepsilon}$, since at least formally

$$
\tilde{f}^{\varepsilon}-f^{\varepsilon}=\left(\tilde{f}^{\varepsilon}-\left(f+\varepsilon f^{1}\right)\right)-\left(\varepsilon^{2} f^{2}+\ldots\right)=\mathcal{O}\left(\varepsilon^{2}\right) .
$$

A delicate point is how to pick the extension of $\tilde{Q}^{1}$. Naturally, the global collision operator $Q+\varepsilon Q^{1}$, and also the first order correction $Q^{1}$, should dissipate the entropy

$$
\left(Q^{1}(f), f\right)_{L_{F}^{2}} \leq 0, \quad \forall f .
$$


In particular, for any smooth functions $g \in \operatorname{ker} \mathcal{T}, h \in \operatorname{ker}\langle\cdot\rangle$ and any $\delta>0$ we have

$$
\left(Q^{1}(g+\delta h), g+\delta h\right)_{L_{F}^{2}} \leq 0
$$

Recall that one of the properties of $\tilde{Q}^{1}$ was that $\left(\tilde{Q}^{1}(g), g\right)_{L_{F}^{2}}=0$ and therefore (67) becomes

$$
\left(\tilde{Q}^{1}(g), h\right)_{L_{F}^{2}}+\left(g, Q^{1}(h)\right)_{L_{F}^{2}}+\delta\left(Q^{1}(h), h\right)_{L_{F}^{2}} \leq 0
$$

which is equivalent to

$$
\left(\tilde{Q}^{1}(g), h\right)_{L_{F}^{2}}+\left(g, Q^{1}(h)\right)_{L_{F}^{2}}=0, \quad\left(Q^{1}(h), h\right)_{L_{F}^{2}} \leq 0, g \in \operatorname{ker} \mathcal{T}, h \in \operatorname{ker}\langle\cdot\rangle .
$$

Recall also that $\tilde{Q}^{1}$ maps constant functions along the flow of $b^{0}$ to zero average functions. We will construct the extension of $\tilde{Q}^{1}$ such that $Q^{1}$ maps zero average functions to constant functions along the flow of $b^{0}$. Notice that in this case the inequality $\left(Q^{1}(h), h\right)_{L_{F}^{2}} \leq 0$ holds true for any $h \in \operatorname{ker}\langle\cdot\rangle$ (actually it becomes equality, by the orthogonality between $\operatorname{ker} \mathcal{T}$ and $\operatorname{ker}\langle\cdot\rangle)$ and that the first condition in (68) uniquely defines $Q^{1}(h) \in \operatorname{ker} \mathcal{T}$ for any $h \in \operatorname{ker}\langle\cdot\rangle$. Moreover, the above construction will ensure the global mass conservation since $\tilde{Q}^{1}$ satisfies the local mass conservation and for any smooth zero average function $h$ we can write, recalling that $\tilde{Q}^{1}(F)=0$

$$
\int_{\mathbb{R}^{2}} \int_{\mathbb{R}^{2}} Q^{1}(h)(x, v) \mathrm{d} v \mathrm{~d} x=\left(Q^{1}(h), F\right)_{L_{F}^{2}}=-\left(h, \tilde{Q}^{1}(F)\right)_{L_{F}^{2}}=0 .
$$

Proposition 4.4 For any smooth functions $g \in \operatorname{ker} \mathcal{T}, h \in \operatorname{ker}\langle\cdot\rangle$ we have

$$
\left(\tilde{Q}^{1}(g), h\right)_{L_{F}^{2}}=-\frac{1}{\tau}(g, K(h))_{L_{F}^{2}}
$$

where

$$
\begin{aligned}
K(h) & =\int_{\mathbb{R}^{2}}\left(s_{1}-\frac{|v|}{\left|v^{\prime}\right|} s_{2}\right) M(v)^{\perp} v^{\prime} \cdot \nabla_{x}\left(\frac{h\left(x, v^{\prime}\right)}{\omega_{c}(x)}\right) \mathrm{d} v^{\prime} \\
& -\int_{\mathbb{R}^{2}} s_{2} \frac{m}{\theta}\left[|v| M(v) \frac{v^{\prime} \cdot v_{\wedge}}{\left|v^{\prime}\right|} h\left(x, v^{\prime}\right)-\left|v^{\prime}\right| M\left(v^{\prime}\right) \frac{v \cdot v_{\wedge}}{|v|} h(x, v)\right] \mathrm{d} v^{\prime}
\end{aligned}
$$

In particular the unique function $Q^{1}(h) \in \operatorname{ker} \mathcal{T}$ satisfying

$$
\left(\tilde{Q}^{1}(g), h\right)_{L_{F}^{2}}+\left(g, Q^{1}(h)\right)_{L_{F}^{2}}=0, \forall g \in \operatorname{ker} \mathcal{T}
$$

is given by

$$
\begin{aligned}
Q^{1}(h) & =\frac{1}{\tau} \int_{\mathbb{R}^{2}}\left(s_{1}-\frac{|v|}{\left|v^{\prime}\right|} s_{2}\right) M(v) \operatorname{div}_{x}\left(\frac{\left\langle{ }^{\perp} v^{\prime} h\right\rangle\left(x, v^{\prime}\right)}{\omega_{c}(x)}\right) \mathrm{d} v^{\prime} \\
& -\frac{1}{\tau} \int_{\mathbb{R}^{2}} s_{2} \frac{m}{\theta}\left[|v| M(v) v_{\wedge} \cdot \frac{\left\langle v^{\prime} h\right\rangle\left(x, v^{\prime}\right)}{\left|v^{\prime}\right|}-\left|v^{\prime}\right| M\left(v^{\prime}\right) v_{\wedge} \cdot \frac{\langle v h\rangle(x, v)}{|v|}\right] \mathrm{d} v^{\prime} .
\end{aligned}
$$

Proof. By Proposition 3.15 we know that

$$
\begin{aligned}
\tilde{Q}^{1}(g) & =\frac{1}{\tau} \int_{\mathbb{R}^{2}}\left(s_{1}-\frac{\left|v^{\prime}\right|}{|v|} s_{2}\right) M(v) F\left(x, v^{\prime}\right) \frac{{ }^{\prime} v}{\omega_{c}(x)} \cdot \nabla_{x}\left(\frac{g\left(x, v^{\prime}\right)}{F\left(x, v^{\prime}\right)}\right) \mathrm{d} v^{\prime} \\
& +\frac{1}{\tau} \int_{\mathbb{R}^{2}} \frac{\left|v^{\prime}\right|}{|v|} s_{2} \frac{m}{\theta}\left(v \cdot v_{\wedge}\right) M(v) F\left(x, v^{\prime}\right)\left[\frac{g\left(x, v^{\prime}\right)}{F\left(x, v^{\prime}\right)}-\frac{g(x, v)}{F(x, v)}\right] \mathrm{d} v^{\prime} .
\end{aligned}
$$


Integrating by parts with respect to $x$ leads to

$$
\begin{aligned}
& \tau\left(\tilde{Q}^{1}(g), h\right)_{L_{F}^{2}}=-\int_{\mathbb{R}^{2}} \int_{\mathbb{R}^{2}} \int_{\mathbb{R}^{2}}\left(s_{1}-\frac{\left|v^{\prime}\right|}{|v|} s_{2}\right) M\left(v^{\prime}\right)^{\perp} v \cdot \nabla_{x}\left(\frac{h(x, v)}{\omega_{c}(x)}\right) \frac{g\left(x, v^{\prime}\right)}{F\left(x, v^{\prime}\right)} \mathrm{d} v^{\prime} \mathrm{d} v \mathrm{~d} x \\
+ & \int_{\mathbb{R}^{2}} \int_{\mathbb{R}^{2}} \int_{\mathbb{R}^{2}} \frac{\left|v^{\prime}\right|}{|v|} s_{2} \frac{m}{\theta}\left(v \cdot v_{\wedge}\right)\left\{M(v) g\left(x, v^{\prime}\right)-M\left(v^{\prime}\right) g(x, v)\right\} \frac{h(x, v)}{F(x, v)} \mathrm{d} v^{\prime} \mathrm{d} v \mathrm{~d} x \\
= & -(g, K(h))_{L_{F}^{2}} .
\end{aligned}
$$

Since

$$
\tau\left(g, Q^{1}(h)\right)_{L_{F}^{2}}=-\tau\left(\tilde{Q}^{1}(g), h\right)_{L_{F}^{2}}=(g, K(h))_{L_{F}^{2}}=(g,\langle K(h)\rangle)_{L_{F}^{2}}
$$

and $Q^{1}(h) \in \operatorname{ker} \mathcal{T}$, we deduce that $Q^{1}(h)=\langle K(h)\rangle / \tau$. For further transformations of the terms in $K(h)$ we need the easy lemma

Lemma 4.1 Let $k=k\left(v, v^{\prime}\right)$ be a bounded function depending only on $r=|v|$ and $r^{\prime}=$ $\left|v^{\prime}\right|$. Then for any function $f=f(x, v) \in L_{F}^{2}$ we have $\int_{\mathbb{R}^{2}} k\left(v, v^{\prime}\right) M(v) f\left(x, v^{\prime}\right) \mathrm{d} v^{\prime} \in L_{F}^{2}$, $\int_{\mathbb{R}^{2}} k\left(v, v^{\prime}\right) M\left(v^{\prime}\right) f(x, v) \mathrm{d} v^{\prime} \in L_{F}^{2}$ and

$$
\begin{aligned}
& \left\langle\int_{\mathbb{R}^{2}} k\left(v, v^{\prime}\right) M(v) f\left(x, v^{\prime}\right) \mathrm{d} v^{\prime}\right\rangle=\int_{\mathbb{R}^{2}} k\left(v, v^{\prime}\right) M(v)\langle f\rangle\left(x, v^{\prime}\right) \mathrm{d} v^{\prime} \\
& \left\langle\int_{\mathbb{R}^{2}} k\left(v, v^{\prime}\right) M\left(v^{\prime}\right) f(x, v) \mathrm{d} v^{\prime}\right\rangle=\int_{\mathbb{R}^{2}} k\left(v, v^{\prime}\right) M\left(v^{\prime}\right)\langle f\rangle(x, v) \mathrm{d} v^{\prime} .
\end{aligned}
$$

Proof. Observe that $\int_{\mathbb{R}^{2}} k\left(v, v^{\prime}\right) M(v)\langle f\rangle\left(x, v^{\prime}\right) \mathrm{d} v^{\prime}, \int_{\mathbb{R}^{2}} k\left(v, v^{\prime}\right) M\left(v^{\prime}\right)\langle f\rangle(x, v) \mathrm{d} v^{\prime}$ depend only on $x$ and $|v|$ and therefore they belong to $\operatorname{ker} \mathcal{T}$. Pick now $\varphi=\varphi(x, v) \in L_{F}^{2}$ a constant function along the flow of $b^{0}$. We can write, observing that $\left(x, v^{\prime}\right) \rightarrow \int_{\mathbb{R}^{2}} k\left(v, v^{\prime}\right) M(v) \varphi(x, v) \mathrm{d} v \in$ $\operatorname{ker} \mathcal{T}$

$$
\begin{aligned}
\int_{\mathbb{R}^{4}} \varphi \int_{\mathbb{R}^{2}} k\left(v, v^{\prime}\right) M(v) f\left(x, v^{\prime}\right) \mathrm{d} v^{\prime} \mathrm{d} v \mathrm{~d} x & =\int_{\mathbb{R}^{2}} \int_{\mathbb{R}^{2}} f\left(x, v^{\prime}\right) \int_{\mathbb{R}^{2}} k\left(v, v^{\prime}\right) M(v) \varphi(x, v) \mathrm{d} v \mathrm{~d} v^{\prime} \mathrm{d} x \\
& =\int_{\mathbb{R}^{2}} \int_{\mathbb{R}^{2}}\langle f\rangle\left(x, v^{\prime}\right) \int_{\mathbb{R}^{2}} k\left(v, v^{\prime}\right) M(v) \varphi(x, v) \mathrm{d} v \mathrm{~d} v^{\prime} \mathrm{d} x \\
& =\int_{\mathbb{R}^{4}} \varphi(x, v) \int_{\mathbb{R}^{2}} k\left(v, v^{\prime}\right) M(v)\langle f\rangle\left(x, v^{\prime}\right) \mathrm{d} v^{\prime} \mathrm{d} v \mathrm{~d} x
\end{aligned}
$$

saying that $\left\langle\int_{\mathbb{R}^{2}} k\left(v, v^{\prime}\right) M(v) f\left(x, v^{\prime}\right) \mathrm{d} v^{\prime}\right\rangle=\int_{\mathbb{R}^{2}} k\left(v, v^{\prime}\right) M(v)\langle f\rangle\left(x, v^{\prime}\right) \mathrm{d} v^{\prime}$. The second statement follows immediately since $\int_{\mathbb{R}^{2}} k\left(v, v^{\prime}\right) M\left(v^{\prime}\right) \mathrm{d} v^{\prime} \in \operatorname{ker} \mathcal{T}$ and thus

$$
\left\langle\int_{\mathbb{R}^{2}} k\left(v, v^{\prime}\right) M\left(v^{\prime}\right) f(x, v) \mathrm{d} v^{\prime}\right\rangle=\int_{\mathbb{R}^{2}} k\left(v, v^{\prime}\right) M\left(v^{\prime}\right) \mathrm{d} v^{\prime}\langle f\rangle(x, v) .
$$

Appealing to Lemma 4.1 and Remark 3.4 we obtain

$$
\begin{aligned}
& \left\langle\int_{\mathbb{R}^{2}}\left(s_{1}-\frac{|v|}{\left|v^{\prime}\right|} s_{2}\right) M(v) \operatorname{div}_{x}\left(\frac{{ }^{\perp} v^{\prime} h\left(x, v^{\prime}\right)}{\omega_{c}(x)}\right) \mathrm{d} v^{\prime}\right\rangle \\
& =\int_{\mathbb{R}^{2}}\left(s_{1}-\frac{|v|}{\left|v^{\prime}\right|} s_{2}\right) M(v) \operatorname{div}_{x}\left\langle\frac{{ }^{\perp} v^{\prime} h\left(x, v^{\prime}\right)}{\omega_{c}(x)}\right\rangle \mathrm{d} v^{\prime}
\end{aligned}
$$

and

$$
\begin{aligned}
& \left\langle\int_{\mathbb{R}^{2}} s_{2} \frac{m}{\theta}\left[|v| M(v)\left(\frac{v^{\prime}}{\left|v^{\prime}\right|} \cdot v_{\wedge}\right) h\left(x, v^{\prime}\right)-\left|v^{\prime}\right| M\left(v^{\prime}\right)\left(\frac{v}{|v|} \cdot v_{\wedge}\right) h(x, v)\right] \mathrm{d} v^{\prime}\right\rangle \\
& =\int_{\mathbb{R}^{2}} s_{2} \frac{m}{\theta}\left[|v| M(v) v_{\wedge} \cdot\left\langle\frac{v^{\prime}}{\left|v^{\prime}\right|} h\right\rangle\left(x, v^{\prime}\right)-\left|v^{\prime}\right| M\left(v^{\prime}\right) v_{\wedge} \cdot\left\langle\frac{v}{|v|} h\right\rangle(x, v)\right] \mathrm{d} v^{\prime}
\end{aligned}
$$


implying that

$$
\begin{aligned}
Q^{1}(h) & =\frac{1}{\tau} \int_{\mathbb{R}^{2}}\left(s_{1}-\frac{|v|}{\left|v^{\prime}\right|} s_{2}\right) M(v) \operatorname{div}_{x}\left(\frac{\left\langle{ }^{\perp} v^{\prime} h\right\rangle\left(x, v^{\prime}\right)}{\omega_{c}(x)}\right) \mathrm{d} v^{\prime} \\
& -\frac{1}{\tau} \int_{\mathbb{R}^{2}} s_{2} \frac{m}{\theta}\left[|v| M(v) v_{\wedge} \cdot \frac{\left\langle v^{\prime} h\right\rangle\left(x, v^{\prime}\right)}{\left|v^{\prime}\right|}-\left|v^{\prime}\right| M\left(v^{\prime}\right) v_{\wedge} \cdot \frac{\langle v h\rangle(x, v)}{|v|}\right] \mathrm{d} v^{\prime} .
\end{aligned}
$$

Corollary 4.1 The extension $Q^{1}$ of the operator $\tilde{Q}^{1}$ satisfying (69) is given by

$$
\begin{aligned}
Q^{1}(f) & =\frac{1}{\tau} \int_{\mathbb{R}^{2}}\left(s_{1}-\frac{\left|v^{\prime}\right|}{|v|} s_{2}\right) M(v) v \cdot\left(\frac{m v_{\wedge}}{\theta}\langle f\rangle\left(x, v^{\prime}\right)-\frac{{ }^{\perp} \nabla_{x}\langle f\rangle\left(x, v^{\prime}\right)}{\omega_{c}(x)}\right) \mathrm{d} v^{\prime} \\
& +\frac{1}{\tau} \int_{\mathbb{R}^{2}} s_{2} \frac{\left|v^{\prime}\right|}{|v|} \frac{m}{\theta}\left(v \cdot v_{\wedge}\right)\left\{M(v)\langle f\rangle\left(x, v^{\prime}\right)-M\left(v^{\prime}\right)\langle f\rangle(x, v)\right\} \mathrm{d} v^{\prime} \\
& +\frac{1}{\tau} \int_{\mathbb{R}^{2}}\left(s_{1}-\frac{|v|}{\left|v^{\prime}\right|} s_{2}\right) M(v) \operatorname{div}_{x}\left(\frac{\left\langle{ }^{\perp} v^{\prime} f\right\rangle\left(x, v^{\prime}\right)}{\omega_{c}(x)}\right) \mathrm{d} v^{\prime} \\
& -\frac{1}{\tau} \int_{\mathbb{R}^{2}} s_{2} \frac{m}{\theta} v_{\wedge} \cdot\left[|v| M(v) \frac{\left\langle v^{\prime} f\right\rangle\left(x, v^{\prime}\right)}{\left|v^{\prime}\right|}-\left|v^{\prime}\right| M\left(v^{\prime}\right) \frac{\langle v f\rangle(x, v)}{|v|}\right] \mathrm{d} v^{\prime}
\end{aligned}
$$

and

$$
\int_{\mathbb{R}^{2}} Q^{1}(f) \mathrm{d} v=-\operatorname{div}_{x} \int_{\mathbb{R}^{2}} v_{\mathrm{QD}} f \mathrm{~d} v, \quad v_{\mathrm{QD}}=-\frac{1}{\tau} \int_{\mathbb{R}^{2}}\left(s_{1}-\frac{\left|v^{\prime}\right|}{|v|} s_{2}\right) M\left(v^{\prime}\right) \mathrm{d} v^{\prime} \frac{{ }^{\prime} v}{\omega_{c}(x)} .
$$

Moreover, when the scattering cross section is constant we have

$$
Q^{1}(f)=\frac{s}{\tau} M(v)\left[\operatorname{div}_{x}\left(\frac{{ }^{\perp} j_{f}(x)}{\omega_{c}(x)}\right)+\frac{\rho_{F}(x)}{\omega_{c}(x)}{ }^{\perp} v \cdot \nabla_{x}\left(\frac{\rho_{f}(x)}{\rho_{F}(x)}\right)\right]
$$

and

$$
\int_{\mathbb{R}^{2}} Q^{1}(f) \mathrm{d} v=-\operatorname{div}_{x} \int_{\mathbb{R}^{2}} v_{\mathrm{QD}} f \mathrm{~d} v, v_{\mathrm{QD}}=-\frac{s}{\tau} \frac{{ }^{\perp} v}{\omega_{c}(x)}
$$

where $\rho_{f}=\int_{\mathbb{R}^{2}} f \mathrm{~d} v^{\prime}, \rho_{F}=\int_{\mathbb{R}^{2}} F \mathrm{~d} v^{\prime}, j_{f}=\int_{\mathbb{R}^{2}} v^{\prime} f \mathrm{~d} v^{\prime}$.

Proof. Using the decomposition $f=\langle f\rangle+(f-\langle f\rangle)$, with $\langle f\rangle \in \operatorname{ker} \mathcal{T}$ and $f-\langle f\rangle \in \operatorname{ker}\langle\cdot\rangle$ and taking into account that $\langle v(f-\langle f\rangle)\rangle=\langle v f\rangle$ we can write

$$
Q^{1}(f)=\tilde{Q}^{1}(\langle f\rangle)+Q^{1}(f-\langle f\rangle)
$$

leading to the formula (70). In particular, when the scattering cross section is constant, we have $s_{1}=s, s_{2}=0$ and (70) reduces to (71).

Remark 4.1 Replacing the vector field $\mathcal{C}$ by (39), the collision operator $Q$ by (3) and the extension operator $Q^{1}$ by (71) in the equation (66) leads to the second order model stated in Theorem 2.3 (case of constant scattering cross section).

It is easily seen, at least for constant scattering cross section, that the kernel of $Q+\varepsilon Q^{1}$ reduces to $\mathbb{R} F=\{\lambda F: \lambda \in \mathbb{R}\}$. Let us establish this property in the general case.

Proposition 4.5 For any $\varepsilon>0$ the only functions in the kernel of $Q+\varepsilon Q^{1}$ are those proportional to $F$. 
Proof. Let $f$ be such that $\left(Q+\varepsilon Q^{1}\right)(f)=0$. Based on the properties of $Q$ and $Q^{1}$ we have

$$
(Q(f), f)_{L_{F}^{2}}=\left(Q(f)+\varepsilon Q^{1}(f), f\right)_{L_{F}^{2}}=0
$$

and

$$
(Q(f), f)_{L_{F}^{2}}=-\frac{1}{2 \tau} \int_{\mathbb{R}^{2}} \int_{\mathbb{R}^{2}} \int_{\mathbb{R}^{2}} s\left(v, v^{\prime}\right) M(v) F\left(x, v^{\prime}\right)\left(\frac{f\left(x, v^{\prime}\right)}{F\left(x, v^{\prime}\right)}-\frac{f(x, v)}{F(x, v)}\right)^{2} \mathrm{~d} v^{\prime} \mathrm{d} v \mathrm{~d} x
$$

implying that $f(x, v)=\rho(x) M(v)$ for some function $\rho=\rho(x)$. Therefore $Q(f)=0$ and thus $Q^{1}(f)=0$. It is easily seen that for $f(x, v)=\rho(x) M(v)$ the formula (70) reduces to

$$
Q^{1}(f)=\frac{1}{\tau} M(v) \frac{v}{|v|} \cdot \int_{\mathbb{R}^{2}}\left(\frac{m v_{\wedge}}{\theta} \rho(x)-\frac{\perp \nabla_{x} \rho}{\omega_{c}(x)}\right)\left(|v| s_{1}-\left|v^{\prime}\right| s_{2}\right) M\left(v^{\prime}\right) \mathrm{d} v^{\prime} .
$$

We are ready if we prove that there is $r>0$ such that

$$
J(v):=\int_{\mathbb{R}^{2}}\left(|v| s_{1}-\left|v^{\prime}\right| s_{2}\right) M\left(v^{\prime}\right) \mathrm{d} v^{\prime}>0,|v|=r
$$

since in that case we deduce that

$$
-\frac{\rho_{F}(x)}{\omega_{c}(x)}{ }^{\perp} \nabla_{x}\left(\frac{\rho(x)}{\rho_{F}(x)}\right)=\frac{m v_{\wedge}}{\theta} \rho(x)-\frac{{ }^{\perp} \nabla_{x} \rho}{\omega_{c}(x)}=0
$$

saying that $\rho=C \rho_{F}$ for some constant $C$, or $f(x, v)=C \rho_{F}(x) M(v)=C F(x, v)$. Recall that $s_{1}, s_{2}$ depend only on $r=|v|, r^{\prime}=\left|v^{\prime}\right|$ and

$$
s_{1}=\frac{1}{2 \pi} \int_{0}^{2 \pi} \sigma(\sqrt{\chi}) \mathrm{d} \alpha, \quad s_{2}=\frac{1}{2 \pi} \int_{0}^{2 \pi} \sigma(\sqrt{\chi}) \cos \alpha \mathrm{d} \alpha, \quad \chi=r^{2}+\left(r^{\prime}\right)^{2}-2 r r^{\prime} \cos \alpha .
$$

Therefore one gets

$$
J(r)=\int_{\mathbb{R}_{+}} \int_{0}^{2 \pi}\left(r-r^{\prime} \cos \alpha\right) \sigma(\sqrt{\chi}) \mu\left(r^{\prime}\right) \mathrm{d} \alpha \mathrm{d} r^{\prime}=\frac{1}{2} \int_{\mathbb{R}_{+}} \int_{0}^{2 \pi} \frac{\partial \chi}{\partial r} \sigma(\sqrt{\chi}) \mu\left(r^{\prime}\right) \mathrm{d} \alpha \mathrm{d} r^{\prime}
$$

where $\mu\left(r^{\prime}\right)=m /(2 \pi \theta) \exp \left(-\frac{m\left(r^{\prime}\right)^{2}}{2 \theta}\right)$. Integrating with respect to $r \in \mathbb{R}_{+}$yields

$$
\int_{\mathbb{R}_{+}} J(r) \mathrm{d} r=\frac{1}{2} \int_{\mathbb{R}_{+}} \int_{0}^{2 \pi} \mu\left(r^{\prime}\right) \int_{\left(r^{\prime}\right)^{2}}^{+\infty} \sigma(\sqrt{\chi}) \mathrm{d} \chi \mathrm{d} \alpha \mathrm{d} r^{\prime}>0 .
$$

Therefore there is $r^{\star}>0$ such that $J\left(r^{\star}\right)>0$.

Another point to be discussed is related to the continuity equation. Since for any $\varepsilon>0$ the solution $f^{\varepsilon}$ of the linear Boltzmann equation (7) satisfies an equation like

$$
\partial_{t} \int_{\mathbb{R}^{2}} f^{\varepsilon} \mathrm{d} v+\operatorname{div} x \int_{\mathbb{R}^{2}} v f^{\varepsilon} \mathrm{d} v=0
$$

we should retrieve a similar one when integrating the second order approximation (66) with respect to $v \in \mathbb{R}^{2}$. We obtain

$$
\partial_{t} \int_{\mathbb{R}^{2}} \tilde{f}^{\varepsilon} \mathrm{d} v+\varepsilon \operatorname{div} \int_{\mathbb{R}^{2}}\left(v_{\wedge}+v_{\mathrm{GD}}\right) \tilde{f}^{\varepsilon} \mathrm{d} v=\varepsilon \int_{\mathbb{R}^{2}} Q^{1}\left(\tilde{f}^{\varepsilon}\right) \mathrm{d} v=-\varepsilon \operatorname{div}_{x} \int_{\mathbb{R}^{2}} v_{\mathrm{QD}} \tilde{f}^{\varepsilon} \mathrm{d} v
$$

and the continuity equation accounts for a new drift $v_{\mathrm{QD}}$, orthogonal to the magnetic lines, due to collisions (cf. Corollary 4.1)

$\partial_{t} \int_{\mathbb{R}^{2}} \tilde{f}^{\varepsilon} \mathrm{d} v+\varepsilon \operatorname{div}_{x} \int_{\mathbb{R}^{2}}\left(v_{\wedge}+v_{\mathrm{GD}}+v_{\mathrm{QD}}\right) \tilde{f}^{\varepsilon} \mathrm{d} v=0, \quad v_{\mathrm{QD}}=-\frac{1}{\tau} \int_{\mathbb{R}^{2}}\left(s_{1}-\frac{\left|v^{\prime}\right|}{|v|} s_{2}\right) M\left(v^{\prime}\right) \mathrm{d} v^{\prime} \frac{{ }^{\prime} v}{\omega_{c}(x)}$.

The previous arguments provide the second order approximation model, as stated by Theorem

2.3. To simplify the presentation we assume here that the scattering cross section is constant. 
Proof. (of Theorem 2.3) Consider $f$ the solution of the problem $\partial_{t} f=Q(f), t>0$ with the initial condition $f(0)=f^{\text {in }}$ and $g^{1}$ the solution of the problem

$$
\partial_{t} g^{1}+\left\langle a \cdot \nabla_{x, v} h^{1}\right\rangle=Q\left(g^{1}\right), \quad t>0, g^{1}(0)=0
$$

where $h^{1}=-\tilde{\mathcal{B}} \cdot \nabla_{x, v} f$. Notice that $\mathcal{T} g^{1}=0,\left\langle h^{1}\right\rangle=0$ and by the previous computations we know that

$$
\partial_{t} f+a \cdot \nabla_{x, v} f+\mathcal{T} f^{1}=Q(f), \quad f^{1}=g^{1}+h^{1} .
$$

We take also a smooth function $f^{2}$ such that

$$
\partial_{t} f^{1}+a \cdot \nabla_{x, v} f^{1}+\mathcal{T} f^{2}=Q\left(f^{1}\right)
$$

which is possible thanks to the evolution equation satisfied by $g^{1}$. As before we write

$$
\left(\partial_{t}+a \cdot \nabla_{x, v}+\varepsilon^{-1} \mathcal{T}\right)\left(f+\varepsilon f^{1}+\varepsilon^{2} f^{2}\right)=Q\left(f+\varepsilon f^{1}+\varepsilon^{2} f^{2}\right)+\varepsilon^{2}\left(\partial_{t}+a \cdot \nabla_{x, v}-Q\right)\left(f^{2}\right) .
$$

Combining to the equation satisfied by $f^{\varepsilon}$ we obtain, with the notation $r^{\varepsilon}=f^{\varepsilon}-\left(f+\varepsilon f^{1}+\right.$ $\left.\varepsilon^{2} f^{2}\right)$

$$
\left(\partial_{t}+a \cdot \nabla_{x, v}+\varepsilon^{-1} \mathcal{T}\right) r^{\varepsilon}=Q\left(r^{\varepsilon}\right)+\varepsilon^{2}\left(Q-\partial_{t}-a \cdot \nabla_{x, v}\right) f^{2} .
$$

Using the entropy inequality we deduce that, for some constant $C_{T}$

$$
\frac{1}{2} \frac{\mathrm{d}}{\mathrm{d} t}\left\|r^{\varepsilon}\right\|_{L_{F}^{2}}^{2} \leq C_{T} \varepsilon^{2}\left\|r^{\varepsilon}\right\|_{L_{F}^{2}}, \quad t \in[0, T], \quad \varepsilon>0
$$

implying that

$$
\left\|r^{\varepsilon}(t)\right\|_{L_{F}^{2}} \leq\left\|r^{\varepsilon}(0)\right\|_{L_{F}^{2}}+T C_{T} \varepsilon^{2}, \quad t \in[0, T], \quad \varepsilon>0 .
$$

Notice that

$$
\begin{aligned}
r^{\varepsilon}(0) & =f_{\text {in }}^{\varepsilon}-\left(f(0)+\varepsilon f^{1}(0)+\varepsilon^{2} f^{2}(0)\right)=f_{\text {in }}^{\varepsilon}-\left(f^{\text {in }}+\varepsilon h^{1}(0)\right)+\mathcal{O}\left(\varepsilon^{2}\right) \\
& =f_{\text {in }}^{\varepsilon}-\left(f^{\text {in }}-\varepsilon \tilde{\mathcal{B}} \cdot \nabla_{x, v} f^{\text {in }}\right)+\mathcal{O}\left(\varepsilon^{2}\right)=\mathcal{O}\left(\varepsilon^{2}\right)
\end{aligned}
$$

and therefore

$$
\sup _{0 \leq t \leq T}\left\|f^{\varepsilon}(t)-\left(f(t)+\varepsilon f^{1}(t)\right)\right\|_{L_{F}^{2}}=\mathcal{O}\left(\varepsilon^{2}\right) .
$$

We also know (cf. (64), (65)) that $f+\varepsilon f^{1}$ satisfies

$$
\left(\partial_{t}-\varepsilon \mathcal{C} \cdot \nabla_{x, v}\right)\left(f+\varepsilon f^{1}\right)=\left(Q+\varepsilon Q^{1}\right)\left(f+\varepsilon f^{1}\right)-\varepsilon^{2}\left(\mathcal{C} \cdot \nabla_{x, v} f^{1}+Q^{1}\left(f^{1}\right)\right)
$$

which is a second order perturbation of the equation (66) (or (19)) satisfied by $\tilde{f}^{\varepsilon}$, implying that

$$
\left(\partial_{t}-\varepsilon \mathcal{C} \cdot \nabla_{x, v}\right) \tilde{r}^{\varepsilon}=\left(Q+\varepsilon Q^{1}\right)\left(\tilde{r}^{\varepsilon}\right)+\varepsilon^{2}\left(\mathcal{C} \cdot \nabla_{x, v} f^{1}+Q^{1}\left(f^{1}\right)\right), \quad \tilde{r}^{\varepsilon}=\tilde{f}^{\varepsilon}-\left(f+\varepsilon f^{1}\right) .
$$

Using now the entropy inequality of $Q+\varepsilon Q^{1}$ yields

$$
\frac{1}{2} \frac{\mathrm{d}}{\mathrm{d} t}\left\|\tilde{r}^{\varepsilon}\right\|_{L_{F}^{2}}^{2} \leq \tilde{C}_{T} \varepsilon^{2}\left\|\tilde{r}^{\varepsilon}\right\|_{L_{F}^{2}}, \quad t \in[0, T], \quad \varepsilon>0 .
$$

Taking into account that $\tilde{r}^{\varepsilon}(0)=\tilde{f}^{\varepsilon}(0)-\left(f(0)+\varepsilon h^{1}(0)\right)=\tilde{f}^{\varepsilon}(0)-\left(f^{\text {in }}-\varepsilon \tilde{\mathcal{B}} \cdot \nabla_{x, v} f^{\text {in }}\right)=0$ we obtain

$$
\left\|\tilde{r}^{\varepsilon}(t)\right\|_{L_{F}^{2}} \leq T \tilde{C}_{T} \varepsilon^{2}, \quad t \in[0, T], \quad \varepsilon>0
$$

saying that

$$
\sup _{0 \leq t \leq T}\left\|\tilde{f}^{\varepsilon}(t)-\left(f(t)+\varepsilon f^{1}(t)\right)\right\|_{L_{F}^{2}}=\mathcal{O}\left(\varepsilon^{2}\right) .
$$

Our conclusion follows immediately from (73), (74). 


\section{A Proofs of Propositions 3.5, 3.6}

Proof. (of Proposition 3.5)

1. We show that $y \rightarrow\left(\psi_{0}(y), \ldots, \psi_{m-1}(y)\right)$ is a change of coordinates. Indeed, if $y, \bar{y} \in \mathbb{R}^{m}$ verify $\psi_{i}(y)=\psi_{i}(\bar{y}), i \in\{0,1, \ldots, m-1\}$, then $y, \bar{y}$ belong to the same characteristic. Thus denoting by $y_{0}$ the discontinuity point of $\psi_{0}$ on this characteristic, there are $h, \bar{h} \in\left[0, T_{c}\left(y_{0}\right)\right)$, with $h \leq \bar{h}$ without loss of generality, such that $y=Y\left(h ; y_{0}\right), \bar{y}=Y\left(\bar{h} ; y_{0}\right)$. Integrating $\left(b^{0} \cdot \nabla_{y} \psi_{0}\right)\left(Y\left(s ; y_{0}\right)\right)=I\left(Y\left(s ; y_{0}\right)\right)=I\left(y_{0}\right)$ between $h$ and $\bar{h}$ we obtain

$$
0=\psi_{0}(\bar{y})-\psi_{0}(y)=\psi_{0}\left(Y\left(\bar{h} ; y_{0}\right)\right)-\psi_{0}\left(Y\left(h ; y_{0}\right)\right)=(\bar{h}-h) I\left(y_{0}\right) .
$$

Therefore $h=\bar{h}$ which implies $y=\bar{y}$. We have shown that $y \in \mathbb{R}^{m}$ is uniquely determined by $\psi_{0}(y), \ldots, \psi_{m-1}(y)$. Indeed, $y$ belongs to the characteristic associated to the invariants $\psi_{1}(y), \ldots, \psi_{m-1}(y)$ and, if we denote by $y_{0}$ the discontinuity point of $\psi_{0}$ on this characteristic, we have $y=Y\left(s ; y_{0}\right)$, where the parameter $s \in\left[0, T_{c}(y)\right)$ is determined by

$$
\psi_{0}(y)-\psi_{0}\left(y_{0}\right)=\int_{0}^{s}\left(b^{0} \cdot \nabla_{y} \psi_{0}\right)\left(Y\left(\tau ; y_{0}\right)\right) \mathrm{d} \tau=s I\left(y_{0}\right) .
$$

Finally, without loss of generality we suppose that $\psi_{0}\left(y_{0}\right)=0$ and thus $\psi_{0}(y) \in\left[0, T_{c}\left(y_{0}\right) I\left(y_{0}\right)\right)=$ $\left[0,\left[\psi_{0}\right]\right)=[0, S)$. Clearly the map $y \rightarrow\left(\psi_{0}(y), \ldots, \psi_{m-1}(y)\right)$ is a surjection between $\mathbb{R}^{m}$ and $[0, S) \times D$, which shows 1 .

2. Notice that $\nabla_{y} \psi_{0} \notin \operatorname{span}\left\{\nabla_{y} \psi_{1}, \ldots, \nabla_{y} \psi_{m-1}\right\}$ since $b^{0} \cdot \nabla_{y} \psi_{0} \neq 0$ and $b^{0} \cdot \nabla_{y} \psi_{1}=\ldots=$ $b^{0} \cdot \nabla_{y} \psi_{m-1}=0$. Thus, for any $i \in\{1, \ldots, m-1\}$ there is a unique vector field $b^{i}$ such that

$$
b^{i} \cdot \nabla_{y} \psi_{j}=\delta_{j}^{i}, \quad j \in\{0,1, \ldots, m-1\},
$$

which proves 2 .

Proof. (of Proposition 3.6)

Notice that for any $y \in \mathbb{R}^{m}$ the function

$$
s \rightarrow u(Y(s ; y))=w\left(\psi_{0}(Y(s ; y)), \psi_{1}(y) \ldots, \psi_{m-1}(y)\right)
$$

is continuous on $\mathbb{R}$. In particular this holds true for any discontinuity point $y_{0}$ of $\psi_{0}$. For any $y=Y\left(s ; y_{0}\right), s \in\left(0, T_{c}\left(y_{0}\right)\right)$ we can write

$$
\begin{aligned}
\left(b^{0} \cdot \nabla_{y} u\right)(y) & =\frac{\mathrm{d}}{\mathrm{d} s}\left\{u\left(Y\left(s ; y_{0}\right)\right)\right\}=b^{0} \cdot \nabla_{y} \psi_{0}(y) \partial_{\psi_{0}} w\left(\psi_{0}(y), \psi_{1}\left(y_{0}\right) \ldots, \psi_{m-1}\left(y_{0}\right)\right) \\
& =I(y) \partial_{\psi_{0}} w\left(\psi_{0}(y), \ldots, \psi_{m-1}(y)\right) .
\end{aligned}
$$

It remains to analyze the differentiability around the point $y_{0}$. Without loss of generality we assume that $I>0$. Taking $s>0$ one gets

$$
\begin{aligned}
b^{0} \cdot \nabla_{y} u\left(y_{0}\right) & =\lim _{s \searrow 0} \frac{1}{s}\left[w\left(\psi_{0}\left(Y\left(s ; y_{0}\right)\right), \psi_{1}\left(y_{0}\right), \ldots, \psi_{m-1}\left(y_{0}\right)\right)-w\left(\psi_{0}\left(y_{0}\right), \ldots, \psi_{m-1}\left(y_{0}\right)\right)\right] \\
& =\lim _{s \searrow 0} \frac{1}{s}\left[w\left(\psi_{0}\left(y_{0}\right)+s I, \psi_{1}\left(y_{0}\right), \ldots, \psi_{m-1}\left(y_{0}\right)\right)-w\left(\psi_{0}\left(y_{0}\right), \ldots, \psi_{m-1}\left(y_{0}\right)\right)\right] \\
& =I\left(y_{0}\right) \partial_{\psi_{0}} w_{+}\left(\psi_{0}\left(y_{0}\right), \ldots, \psi_{m-1}\left(y_{0}\right)\right)
\end{aligned}
$$


where $\partial_{\psi_{0}} w_{+}$stands for the right derivative of $w$ with respect to $\psi_{0}$. Taking now $s<0$, using the $S$-periodicity of $w$ with respect to $\psi_{0}$, we obtain

$$
\begin{aligned}
b^{0} \cdot \nabla_{y} u\left(y_{0}\right) & =\lim _{s \nearrow 0} \frac{1}{s}\left[w\left(\psi_{0}\left(Y\left(T_{c}+s ; y_{0}\right)\right), \ldots, \psi_{m-1}\left(y_{0}\right)\right)-w\left(\psi_{0}\left(y_{0}\right), \ldots, \psi_{m-1}\left(y_{0}\right)\right)\right] \\
& =\lim _{s \nearrow 0} \frac{1}{s}\left[w\left(\psi_{0}\left(y_{0}\right)+\left(T_{c}+s\right) I, \ldots, \psi_{m-1}\left(y_{0}\right)\right)-w\left(\psi_{0}\left(y_{0}\right), \ldots, \psi_{m-1}\left(y_{0}\right)\right)\right] \\
& =\lim _{s \nearrow 0} \frac{1}{s}\left[w\left(\psi_{0}\left(y_{0}\right)+s I, \ldots, \psi_{m-1}\left(y_{0}\right)\right)-w\left(\psi_{0}\left(y_{0}\right), \ldots, \psi_{m-1}\left(y_{0}\right)\right)\right] \\
& =I\left(y_{0}\right) \partial_{\psi_{0}} w_{-}\left(\psi_{0}\left(y_{0}\right), \ldots, \psi_{m-1}\left(y_{0}\right)\right)
\end{aligned}
$$

where $\partial_{\psi_{0}} w_{-}$stands for the left derivative of $w$ with respect to $\psi_{0}$. Combining (75), (76) we deduce that $w$ is differentiable with respect to $\psi_{0}$ and at any point $y \in \mathbb{R}^{m}$

$$
b^{0} \cdot \nabla_{y} u=I(y) \partial_{\psi_{0}} w\left(\psi_{0}(y), \ldots, \psi_{m-1}(y)\right) .
$$

Moreover, for any $i \in\{1, \ldots, m-1\}$ we have

$$
b^{i} \cdot \nabla_{y} u=\sum_{j=0}^{m-1} \partial_{\psi_{j}} w b^{i} \cdot \nabla_{y} \psi_{j}=\partial_{\psi_{i}} w\left(\psi_{0}(y), \ldots, \psi_{m-1}(y)\right), \quad y \in \mathbb{R}^{m} .
$$

Acknowledgements. This work was initiated during the visit of the first author at the University of Texas at Austin. The second author acknowledges partial support from NSF grant DMS 1109625. Support from the Institute for Computational Engineering and Sciences at the University of Texas at Austin is also gratefully acknowledged.

\section{References}

[1] N. Ben Abdallah, I.M. Gamba, A. Klar, The Milne problem for high field kinetic equations, SIAM J. Appl. Math. 64(2004) 1709-1736.

[2] N.N. Bogoliubov, Y.A. Mitropolsky, Asymptotic methods in the theory of nonlinear oscillations, Gordon and Breach Sciences Publishers, New York, 1961.

[3] M. Bostan, The Vlasov-Poisson system with strong external magnetic field. Finite Larmor radius regime, Asymptot. Anal. 61(2009) 91-123.

[4] M. Bostan, Transport equations with disparate advection fields. Application to the gyrokinetic models in plasma physics, J. Differential Equations 249(2010) 1620-1663.

[5] M. Bostan, Gyrokinetic Vlasov equation in three dimensional setting. Second order approximation, SIAM J. Multiscale Model. Simul. 8(2010) 1923-1957.

[6] M. Bostan, Transport of charged particles under fast oscillating magnetic fields, SIAM J. Math. Anal. 44(2012) 1415-1447.

[7] M. Bostan, I.M. Gamba, T. Goudon, The linear Boltzmann equation with space periodic electric field, Nonlinear partial differential equations and related topics, Amer. Math. Soc. Transl. Ser. 2 229(2010) 51-66. 
[8] M. Bostan, I.M. Gamba, T. Goudon, A. Vasseur, Boundary value problems for the stationary Vlasov-Boltzmann-Poisson equation, Indiana Univ. Math. J. 59(2010) 16291660 .

[9] M. Bostan, C. Negulescu, Mathematical models for strongly magnetized plasmas with mass disparate particles, Discrete Contin. Dyn. Syst. Ser. B 15(2011) 513-544.

[10] A.J. Brizard, A guiding-center Fokker-Planck collision operator for nonuniform magnetic fields, Phys. Plasmas 11(2004) 4429-4438.

[11] A.J. Brizard, T.S. Hahm, Foundations of nonlinear gyrokinetic theory, Rev. Modern Phys. 79(2007) 421-468.

[12] C. Cercignani, The Boltzmann equation and its applications, Springer-Verlag, New York 1988.

[13] C. Cercignani, I.M. Gamba, C.D. Levermore, High field approximations to a Boltzmann-Poisson system boundary conditions in a semiconductor, Appl. Math. Lett. 10(1997) 111-118.

[14] C. Cercignani, I.M. Gamba, C.D. Levermore, A drift-collision balance asymptotic for a Boltzmann-Poisson system in bounded domains, SIAM J. Appl. Math. 61(2001) 19321958.

[15] C. Cercignani, R. Illner, M. Pulvirenti, The mathematical theory of dilute gases, Springer-Verlag, Berlin 1994.

[16] E. Frénod, E. Sonnendrücker, Homogenization of the Vlasov equation and of the VlasovPoisson system with strong external magnetic field, Asymptotic Anal. 18(1998) 193-213.

[17] X. Garbet, G. Dif-Pradalier, C. Nguyen, Y. Sarazin, V. Grandgirard, Ph. Ghendrih, Neoclassical equilibrium in gyrokinetic simulations, Phys, Plasmas 16(2009).

[18] X. Garbet, Towards a full self-consistent numerical simulation of tokamak plasma turbulence, Plasma Phys. Cont. Fusion 39(1997).

[19] X. Garbet, Turbulence modeling in fusion plasmas, Europhysics News 29(1998).

[20] F. Golse, L. Saint-Raymond, The Vlasov-Poisson system with strong magnetic field, J. Math. Pures Appl. 78(1999) 791-817.

[21] V. Grandgirard, M. Brunetti, P. Bertrand, N. Besse, X. Garbet, P. Ghendrih, G. Manfredi, Y. Sarazin, O. Sauter, E. Sonnendrücker, J. Vaclavik, L. Villard, A drift-kinetic semi-Lagrangian 4D code for ion turbulence simulation, J. Comput. Phys. 217(2006) 395-423.

[22] R.D. Hazeltine, J.D. Meiss, Plasma confinement, Dover Publications, Inc. Mineola, New York, 2003.

[23] R. G. Littlejohn, A guiding center Hamiltonian : A new approach, J. Math. Phys. 20(1979) 2445-2458.

[24] R. G. Littlejohn, Hamiltonian formulation of guiding center motion, Phys. Fluids 24(1981) 1730-1749.

[25] P.A. Markowich, C. Ringhofer, C. Schmeiser, Semiconductor equations, SpringerVerlag, New York 1990. 
[26] F. Poupaud, Runaway phenomena and fluid approximation under high fields in semiconductor kinetic theory, Z. Angew. Math. Mech. 72(1992) 359-372.

[27] J.-M. Rax, Physique des plasmas, Cours et applications, Dunod 2007.

[28] W. Rudin, Principles of Mathematical Analysis, McGraw-Hill 1976.

[29] X.Q. Xu, M.N. Rosenbluth, Numerical simulation of ion-temperature-gradient-driven modes, Phys. Fluids, B 3(1991) 627-643. 